\title{
An experimental study of binary collisions of miscible droplets with non-identical viscosities
}

\author{
Karrar H. Al-Dirawi ${ }^{1}$. Andrew E. Bayly ${ }^{1}$ \\ Received: 21 May 2019 / Revised: 13 December 2019 / Accepted: 24 December 2019 / Published online: 22 January 2020 \\ (c) The Author(s) 2020
}

\begin{abstract}
The dynamics of binary collisions of equi-diameter droplets with non-identical viscosities have been investigated experimentally and compared to previously generated data from identical droplet collisions (Al-Dirawi and Bayly in Phys Fluids 31(2):027105, 2019). Three hydroxypropyl methylcellulose (HPMC) aqueous solutions, $2 \%, 4 \%$, and $8 \%$ HPMC, were used to generate the droplets of different viscosities, 2.8, 8.2, and $28.4 \mathrm{mPa}$ s, respectively. High-speed imaging techniques were applied to observe and capture the collision outcomes. Collision outcomes were characterised and regime maps were generated. The non-identical viscosity droplet collisions produced regime maps with well-defined boundaries which are comparable in shape to the conventional regime maps of identical droplet collisions. The boundaries of the bouncing and reflexive separation regimes of the non-identical collisions show intermediate position between the identical cases of the low and the high viscosity droplets. However, the boundary of the stretching separation regimes of the non-identical collisions showed good agreement with the boundary of the identical case of the lower viscosity droplet. Moreover, the ability of models developed for predicting the regimes boundaries of collisions of identical viscosity droplets was assessed for the non-identical collisions. They proved capable in the non-identical cases, and the changes in adjustable parameters were consistent with the underlying physical basis of the models.
\end{abstract}

Electronic supplementary material The online version of this article (https://doi.org/10.1007/s00348-019-2874-3) contains supplementary material, which is available to authorized users.

Karrar H. Al-Dirawi

karrarchemi.13@gmail.com

$\triangle$ Andrew E. Bayly

a.e.bayly@leeds.ac.uk

1 School of Chemical and Process Engineering, University of Leeds, Leeds LS2 9JT, UK 


\section{Graphic abstract}

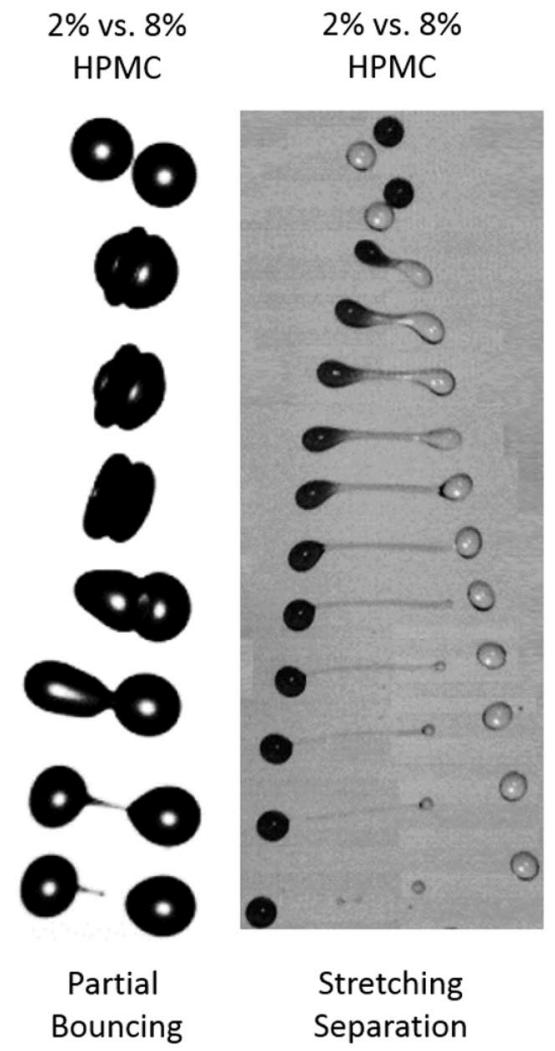

$2 \%$ vs. $4 \%$ HPMC

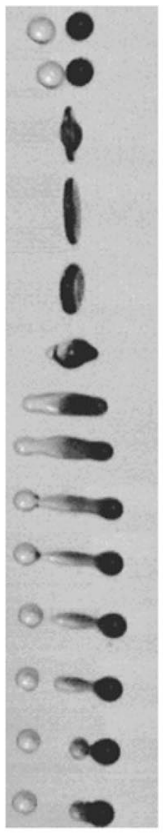

Reflexive

Separation

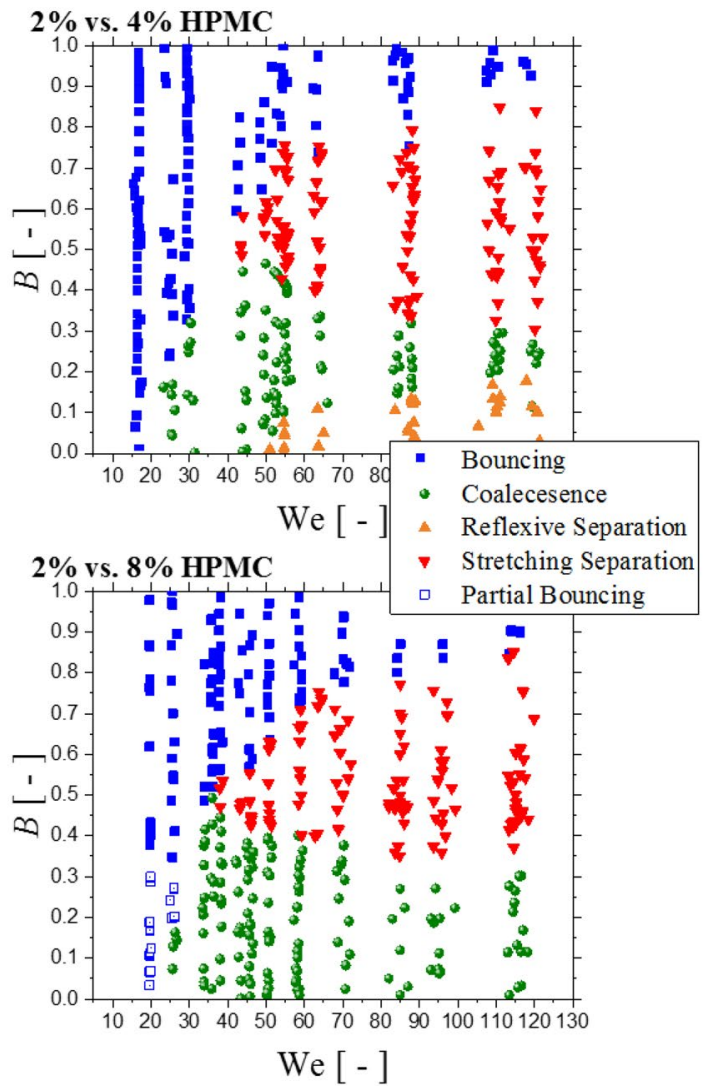

1.1 Binary droplet collisions theory

In the past few decades, extensive research has been conducted to understand the physics that determines the outcome of binary droplet collisions. Reviews of the area are provided by Orme (1997) and Krishnan and Loth (2015). However, the majority of this work concerns collisions between droplets that have identical physical properties, and only a few studies look at collisions between droplets that have non-identical physical properties, e.g., Chen (2007), Chen and Chen (2006), Gao et al. (2005), Focke et al. (2013), Planchette et al. (2010, 2011, 2012, 2017).

To provide the context for the work reported here, this section summarises the collision outcomes and regime maps observed for collisions of droplets with identical viscosities and highlights the effect of viscosity on the collisions. A brief summary of the studies of collisions of droplets with non-identical fluids is then presented which will highlight the gap that motivates this study.

In the literature of binary collisions of droplets that have identical physical properties, five distinct collision outcomes 


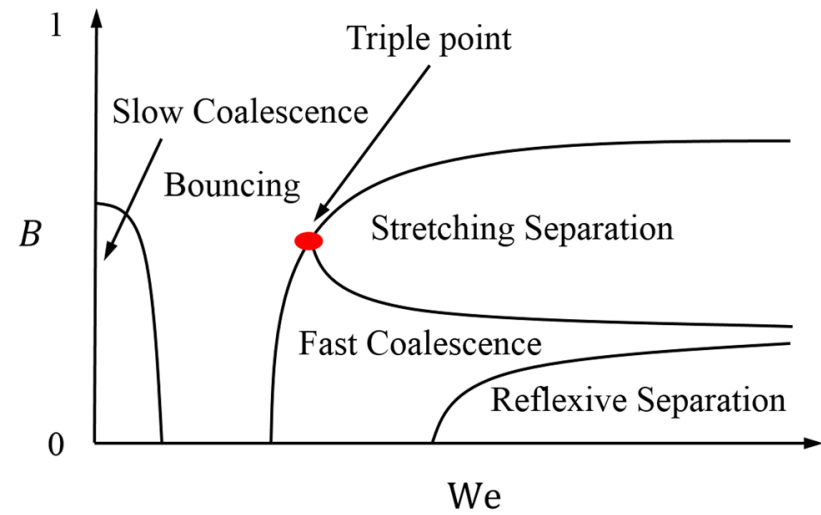

Fig. 1 Schematic illustration of a typical regime map; reproduced from (Al-Dirawi and Bayly 2019), with the permission of AIP Publishing

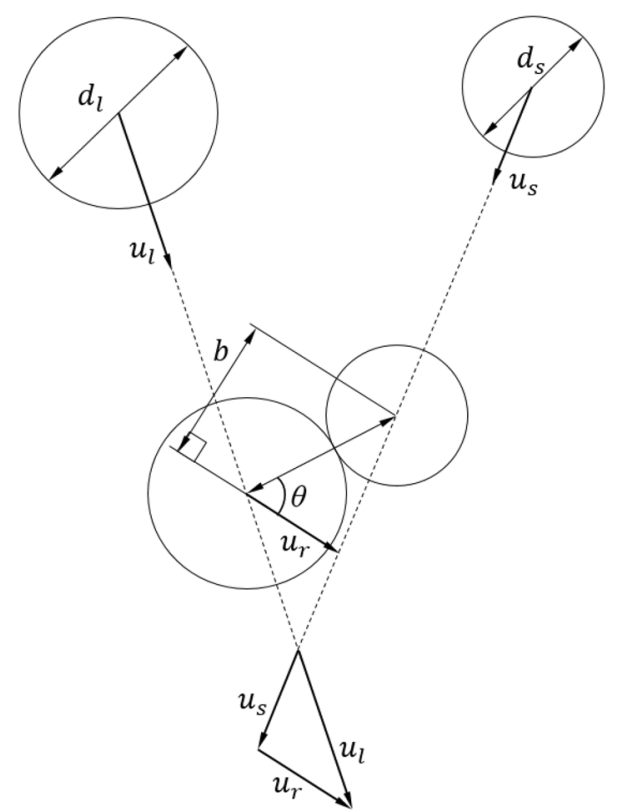

Fig. 2 Schematic of the droplet collision geometry that shows the definition of the impact parameter and the velocities vectors, reproduced from (Al-Dirawi and Bayly 2019), with the permission of AIP Publishing

have been observed: slow coalescence, bouncing, fast coalescence, reflexive separation and stretching separation (Qian and Law 1997). These regimes are mapped in the parameter space of the impact parameter $(B)$ and the Weber number (We), as shown in Fig. 1. The impact parameter represents the offset between the colliding droplets. It is defined by the normal distance $(b)$ from the centre of one of the colliding droplets to the vector of the relative velocity, which is plotted from the centre of the other droplet, normalised by the sum of the two droplets radii, Eq. (1), as sketched in Fig. 2.
Therefore, $B$ has a value between 1 and 0 , where 1 indicates a grazing collision and 0 a head-on collision:

$B=\frac{2 b}{d_{\mathrm{s}}+d_{1}}$,

where $d_{\mathrm{s}}$ and $d_{1}$ are the small and the large droplet diameters, respectively, in the general case of any size ratio between the colliding droplet. The Weber number is the ratio of the kinetic energy to the surface energy and it is given by

$\mathrm{We}=\frac{\rho u_{\mathrm{r}}^{2} d}{\sigma}$,

where $\rho, \sigma$, and $u_{\mathrm{r}}$ are the droplet fluid density, surface tension, and the magnitude of the collision relative velocity, respectively. In the case of non-identical physical properties or diameters, it is necessary to select one of the droplets as a reference on which to base the We. In the case of non-equal diameters, this is often the smaller droplet diameter e.g., Qian and Law (1997); Ashgriz and Poo (1990). (In this work $d_{\mathrm{s}}=d_{1}=d$, as only identical droplet size collisions were studied, additionally, $\rho, \sigma$ were also identical.)

The five distinct collision outcomes are characterised as follows. For given droplet materials and sizes the We can be considered as an indicator of collision velocity or energy.

- Slow coalescence: occurs when the two droplets approach each other at low We, this allows sufficient time for the interfaces to merge without significant deformation.

- Bouncing: occurs at a higher We than slow coalescence, in this case an air film is trapped between the two colliding droplets; this prevents the interfaces from being close enough to each other for the intermolecular forces to merge the droplets. As a result, the droplets deform on impact, their kinetic energy is converted to viscous loss and surface energy, and then the latter is converted back to the kinetic energy of the rebounding droplet and viscous loss as the droplets recover to their equilibrium spherical shape.

- Fast coalescence: with increasing We, the inter-droplet air film thins to a point, where coalescence occurs at a point with significant droplet deformation is observed.

- Reflexive separation: occurs at low $B$ (or at near headon collisions) at We higher than that of fast coalescence. The droplets first merge forming a disc shaped droplet, which is a circular lamella bounded by a toroidal rim at its highest deformation. The effects of surface tension then contract the disc and a reflexive internal flow is induced that is strong enough to form a cylindrical droplet with its axis perpendicular to the 
plane of the disc. This cylinder then breaks up into two or more droplets.

- Stretching separation: starts at a We slightly higher than that of the fast coalescence but at a moderate offset, $B$. The two droplets partially interact and because of the momentum of the non-interacted regions the merged area is stretched forming a ligament between the two colliding droplets that eventually breaks up into satellite droplets.

The reader is also referred to Fig. 4, which shows the dynamics of the last four collision outcomes.

In collisions of identical fluids, the effect of viscosity was first considered by Jiang et al. (1992); however, it was limited to a very narrow range $0.4-3.5 \mathrm{mPa}$ s. Due to the importance of understanding collisions at a higher viscosity range in applications like spray drying, more recent studies have considered viscosities up to $100 \mathrm{mPa}$ s (Willis and Orme 2003; Kuschel and Sommerfeld 2013; Sommerfeld and Kuschel 2016; Finotello et al. 2018b; Gotaas et al. 2007). It has been reported that elevating the viscosity would shift the boundary of reflexive separation toward high Weber numbers, and the stretching separation would drift to higher impact parameters (Gotaas et al. 2007; Kuschel and Sommerfeld 2013).

While more limited, collisions between droplets with non-identical physical properties have also been studied. These studies can be divided into two categories: collisions between immiscible droplets and collisions between miscible droplets. The experimental and theoretical studies of immiscible droplet collisions with non-identical viscosity are more numerous, e.g., (Chen and Chen 2006; Planchette et al. 2010, 2011, 2012, 2017; Tsuru et al. 2010). However, studies of collisions of miscible droplets from unlike fluids are relatively scarce. Chen (2007) and Gao et al. (2005) experimentally investigated water, ethanol and diesel collisions; this limited their viscosities to a low range at relatively low viscosities (1-3.16 mPa s). Focke et al. (2013) made a detailed numerical and experimental study of collisions with a high viscosity ratio (2.6 vs. $60 \mathrm{mPa} \mathrm{s}$ ); however, their study was limited to the coalescence regime with a fixed We of 26 and no regime maps were constructed.

In this work, the role of the viscosity difference between the colliding droplets will be experimentally studied, with collision conditions covering the whole regime map, for miscible fluids at viscosity range of $2.8-29 \mathrm{mPa}$ s. This paper is structured as follows. Section 2 will review the existing models that aim to predict the regime boundaries for identical droplet collisions. Section 3, will describe the experimental methodology and materials used. In Sect. 4, the resulting regime maps will be presented and discussed, and the applicability of the existing models, presented in Sect. 2, to collisions of droplets with non-identical viscosities will be examined.

\section{The existing models}

The prediction of the regime boundaries for droplet collisions of identical fluids, have received substantial attention in the literature and are briefly reviewed here. A composite model to predict the regime boundaries will be proposed to be applied for collisions of miscible droplets with different viscosities.

\subsection{Bouncing}

Estrade et al. (1999) were the first to model the boundary of the bouncing regime for inviscid droplets, Hu et al. (2017) extended this model to allow for viscosity. These models are based on the criteria that bouncing occurs if the impact kinetic energy does not cause a droplet deformation that exceeds a critical deformation limit. This deformation limit is set empirically through a shape factor. Although, Estrade's model has been widely used, it was reported in many studies that it is not accurate especially in predicting the bouncing boundary below the triple point (Kuschel and Sommerfeld 2013; Sommerfeld and Kuschel 2016; Finotello et al. 2018a, b; Al-Dirawi and Bayly 2019). Al-Dirawi and Bayly (2019) suggested several improvements and corrections to the models of Estrade and $\mathrm{Hu}$, for example to the treatment of kinetic energy and the shape factor, and alternatively proposed an improved model for the boundary of the bouncing regime:

$\mathrm{We}_{\mathrm{c}}=\frac{12 \Delta^{2}\left(1+\Delta^{2}\right)\left(1+\Delta^{3}\right)^{2}\left(\phi_{\text {o.s. }}^{\prime \prime}-1\right)}{\left(X s+\Delta^{3} X_{1}\right)\left(1-B^{2}\right)(1-\alpha)}$,

where $\Delta$ is the size ratio $\left(d_{\mathrm{s}} / d_{1}\right)$ in case of collisions between droplets that have different sizes, and $\alpha$ is the viscous loss factor that represents the ratio of the viscous loss energy to the initial impact kinetic energy. The shape factor, $\phi_{\text {o.s. }}^{\prime \prime}$ is defined by

$$
\begin{aligned}
\phi_{\text {o.s. }}^{\prime \prime}= & \frac{1}{\left(2+2 \Delta^{2}\right)}\left(\left(\left(\frac{1}{1-e_{1}}\right)^{\frac{1}{3}}+\frac{1}{2}\left(\frac{\left(1-e_{1}\right)^{\frac{2}{3}}}{e_{1}}\right) \ln \left(\frac{1+e_{1}}{1-e_{1}}\right)\right)\right. \\
& +\Delta^{2}\left(\left(\frac{1}{1-e_{\mathrm{s}}}\right)^{\frac{1}{3}}+\frac{1}{2}\left(\frac{\left(1-e_{\mathrm{s}}\right)^{\frac{2}{3}}}{e_{\mathrm{s}}}\right) \ln \left(\frac{1+e_{\mathrm{s}}}{1-e_{\mathrm{s}}}\right)\right),
\end{aligned}
$$

w h e r e $\quad e_{1}=\left(\left(1-\left(c_{1}^{2} / a_{1}^{2}\right)\right) / 1+\Psi B^{\beta}\right)^{0.5}, \quad$ a n d $e_{\mathrm{s}}=\left(1-\left(c_{\mathrm{s}}^{2} / a_{\mathrm{s}}^{2}\right) / 1+\Psi B^{\beta}\right)^{0.5} . c$ and $a$ represent the major and the minor radii of the spheroidal shape of the droplets at the maximum deformation just before the instant of rebound. The subscripts $l$ and $s$ are used to differentiate between the colliding droplets, as they can be used to set a different deformation limit to each droplet which might occur due to the difference in the size or/and fluid properties. $\Psi$ and $\beta$ are empirical parameters that are used to capture the effect of the shape factor on the deformation limit. $X_{\mathrm{s}}$ and $X_{1}$ represent the 
ratio of the interaction region volume of the colliding droplets, which were first reported by Ashgriz and Poo (1990):

$X_{\mathrm{s}}=\left\{\begin{array}{ll}\left(1-\frac{1}{4 \Delta^{3}}(2 \Delta-\tau)^{2}(\Delta+\tau)\right) & \text { for } h>\frac{d_{\mathrm{s}}}{2} \\ \frac{1}{4 \Delta^{3}} \tau^{2}(3 \Delta-\tau) & \text { for } h \leq \frac{d_{\mathrm{s}}}{2}\end{array}\right.$,

and

$X_{1}=\left\{\begin{array}{ll}1-\frac{1}{4}(2-\tau)^{2}(1+\tau) & \text { for } h>\frac{d_{1}}{2}, \\ \frac{1}{4} \tau^{2}(3-\tau) & \text { for } h \leq \frac{1}{2}\end{array}\right.$,

where

$\tau=(1-B)(1+\Delta)$

and

$h=\frac{1}{2}\left(d_{1}+d_{\mathrm{s}}\right)(1-B)$.

\subsection{Stretching separation}

Both Ashgriz and Poo (1990) and Jiang et al. (1992) modelled the stretching separation boundary. However, the latter was reported to perform better for high viscosity droplet collisions (Kuschel and Sommerfeld 2013; Sommerfeld and Kuschel 2016; Gotaas et al. 2007). This is because the model of Jiang et al. (1992) considers the viscous losses, whereas the model of Ashgriz and Poo (1990) was developed for inviscid fluids. Therefore, the model of Jiang et al. (1992) will be considered in this study.

The model of Jiang et al. (1992) was developed based on momentum conservation, assuming that the united droplets behave as two circular plates that are sliding on each other. The sliding velocity is the component of the relative velocity that is perpendicular to the line between the two droplets centres, while the other component is responsible for the deformation. The resistance to the sliding velocity is the surface tension forces along the circumference of the plates and the viscous loss due to the shearing flow layer between the sliding plates (i.e., the droplets). The model is given by

$B=\frac{1}{\mathrm{We}^{0.5}}\left[1+k \frac{\mu}{\sigma}\left(\frac{\rho d}{\sigma}\right)^{0.5}\right]$,

where $k$ is a constant that can be used to fit the model to the experimental data. However, subsequently, the model has been widely used with two fitting parameters, $C_{a}$ and $C_{b}$, as in Eq. (10) (Kuschel and Sommerfeld 2013; Sommerfeld and Kuschel 2016; Gotaas et al. 2007; Finotello et al. 2017, $2018 \mathrm{a}, \mathrm{b})$. It should be noted that $k$ in Eq. (9) and $C_{b}$ in
Eq. (10) are not dimensionless parameters and have a unit of $\left(\mathrm{m}^{2} \mathrm{~s}^{-2}\right)$, while $C_{a}$ is a dimensionless parameter:

$B=\frac{C_{a}}{\mathrm{We}^{0.5}}\left[1+C_{b} \frac{\mu}{\sigma}\left(\frac{\rho d}{\sigma}\right)^{0.5}\right]$.

Sommerfeld and Pasternak (2019) reported that $C_{b}$ can be fixed and changing only $C_{a}$ can fit the model to experiments. The authors used a wide range of experiments to correlate the optimum $C_{a}$ with Ohnesorge number $(\mathrm{Oh})$ by fixing $C_{b}=1\left(\mathrm{~m}^{2} \mathrm{~s}^{-2}\right)$. Ohnesorge number is given by $\mathrm{Oh}=\mu / \sqrt{\rho \sigma d}$, where $\mu$ and $d$ are the droplet viscosity and diameter, respectively. Two polynomial correlations $\left(C_{a}=f(\mathrm{Oh})\right)$ were reported, one for pure liquids, and the other for solutions. The latter is applicable to this study, as HPMC solutions are used, which is given by

$C_{a}=2.63-7.20 \mathrm{Oh}+7.86 \mathrm{Oh}^{2}+1.40 \mathrm{Oh}^{3}$.

Another approach to predict the boundary of stretching separation was reported by Planchette et al. (2012). The authors discussed that if part of the kinetic energy is converted into rotational energy as reported by Brazier-Smith et al. (1972), there is a need for an effective impact parameter, $B_{\text {eff. }}$. This is because the droplets suffer strong distortion when undergoing off-centre collisions. The set criteria to estimate $B_{\text {eff }}$ are that $B_{\text {eff }}$ tends to $B$ when We tends to 0 and/ or $B$ tends to 1 , whereas $\left(B_{\text {eff }}-B\right)$ increases with We when $B$ tends to 0 . Based on these criteria, $B_{\text {eff }}$ is given by

$B_{\mathrm{eff}}=B+(1-B) \frac{u_{\mathrm{r}}}{U^{*}}$,

where $U^{*}$ is a fitting parameter, which increases monotonically with the viscosity.

Planchette et al. (2012) showed that taking a stretching separation boundary for high viscosity droplets collisions as a reference boundary for $B_{\text {eff }}$, leads to that the boundaries of the lower viscosity droplets collapsing on that boundary using $B_{\text {eff }}$. Hence, a unified boundary can be used to represent systems with different viscosities.

\subsection{Reflexive separation}

Ashgriz and Poo (1990) have reported an inviscid model to predict the boundary of reflexive separation. The model was developed based on an energy balance between the kinetic energy and surface energy. The criterion of the model is that reflexive separation occurs if the combined reflexive kinetic energy based on an elastic collision of the two droplets is greater than $75 \%$ of the surface energy of the coalesced droplet. The model is given by 
$\mathrm{We}_{\mathrm{c}}=3\left[7\left(1+\Delta^{3}\right)^{\frac{2}{3}}-4\left(1+\Delta^{2}\right)\right] \frac{\Delta\left(1+\Delta^{3}\right)^{2}}{\Delta^{6} ¥_{1}+¥_{2}}$,

where

$¥_{1}=2(1-(0.5 B(1+\Delta)))^{2}\left(1-(0.5 B(1+\Delta))^{2}\right)^{\frac{1}{2}}-1$, and

$¥_{2}=2(\Delta-(0.5 B(1+\Delta)))^{2}\left(\Delta^{2}-(0.5 B(1+\Delta))^{2}\right)^{\frac{1}{2}}-\Delta^{3}$.

The above model is only valid for inviscid fluids as the viscous loss is neglected, it has been shown to give good agreement for water and similar viscosity fluids (Sommerfeld and Kuschel 2016). However, Jiang et al. (1992) reported that the onset of reflexive separation at head-on collisions ( $\mathrm{We}_{\mathrm{Fc} / \mathrm{Rs}}$ ) can be linearly correlated with $\mathrm{Oh}$. The Ohnesorge number is often a valuable group for capturing the physics governing the break-up of viscous threads (Ohnesorge 1936; McKinley 2005), and the mechanism which governs reflexive separation. This encouraged many authors to produce empirical correlations $\left(\mathrm{We}_{\mathrm{Fc} / \mathrm{Rs}}=f(\mathrm{Oh})\right)$ to define the onset of reflexive separation at head-on collision. Qian and Law (1997) used droplets of hydrocarbons to produce

$\mathrm{We}_{\mathrm{FC} / \mathrm{RS}}=17+510 \mathrm{Oh}$.

Later, Gotaas et al. (2007) reported that for Oh $>0.04$ the correlation is no longer linear and proposed two correlations:

$\mathrm{We}_{\mathrm{FC} / \mathrm{RS}}=14.8+643.1 \mathrm{Oh}$ for $\mathrm{Oh}<0.04$ and

$\mathrm{We}_{\mathrm{FC} / \mathrm{RS}}=9309 \mathrm{Oh}^{1.7056}$ for $\mathrm{Oh}>0.04$.

Recent studies used these correlations to shift the curve of the model of Ashgriz and Poo (1990), Eq. (13), toward higher We (Sommerfeld and Kuschel 2016; Finotello et al. 2018a, b). However, this approach is not always accurate as the fittings of $\mathrm{We}_{\mathrm{FC} / \mathrm{RS}}=f(\mathrm{Oh})$ correlations show considerable scatter (Sommerfeld and Kuschel 2016; Finotello et al. 2018b)

Hu et al. (2017) used the same approach as Ashgriz and Poo (1990) but with a different definition of collisional kinetic energy for $B>0$. They used a spherical cap geometry to define the interaction volume rather than a prolate volume used by Ashgriz and Poo. They also introduced a viscous loss factor $\alpha_{2}$, defined as the ratio of the viscously dissipated energy to the collisional kinetic energy. This factor is used as a fitting parameter to fit the model with the experimental data. The model is given by
$\mathrm{We}_{\mathrm{c}}=\frac{3\left(7\left(1+\Delta^{3}\right)^{\frac{2}{3}}-4\left(1+\Delta^{2}\right)\right)\left(1+\Delta^{3}\right)^{2}}{\Delta^{2}\left(\left(2-\alpha_{2}\right)\left(\Delta^{3} X_{1}+X_{\mathrm{s}}\right)-\left(1+\Delta^{3}\right)\right)}$,

where $X_{\mathrm{s}}$ and $X_{1}$ are given by Eqs. (5) and (6), respectively.

Recently, Planchette et al. (2017) developed a model to predict the onset of reflexive separation for head-on collisions, i.e., $B=0$. The model was developed based on the analogy between the dynamics of reflexive separation and the compression and relaxation process of liquid springs, and carefully considers the energy losses during these processes. The criterion used for the separation threshold (called fragmentation by the authors) is based on a Rayleigh-like analysis of the break-up of the cylinder (eighth image in the reflexive separation sequence of Fig. 4) formed on contraction of the rimmed lamellar disc. The critical aspect ratio of the cylinder above which separation occurs was determined from experimental data to be $\zeta_{\text {crit }} \geq 3$, for $\mathrm{Oh}<0.1$. Two viscous losses are considered by the model, a loss in the compression period and a loss in the relaxation period. The loss in the compression period is characterised by a loss factor $a$, which is the ratio of the viscous loss in the compression period to the initial kinetic energy of the droplets $\left(\rho \pi d^{3} u_{\mathrm{r}}^{2} / 24\right)$. For a wide range of $\mathrm{Oh}(0.02-0.15)$, the loss factor $a \sim 0.65$; however, for $\mathrm{Oh}<0.02, a$ is lower and for $\mathrm{Oh}>0.15, a$ is higher. In the early time of the relaxation period, a factor, $q$, is proposed to allow for losses. The proposed model of the critical collision velocity is given by

$u_{\mathrm{r}}^{\text {theo }}=0.8 p \zeta_{\text {crit }} \sqrt{\frac{24 \sqrt{2} / \pi}{\left(1-a-q /\left(\mathrm{We}^{0.5} \mathrm{Oh}\right)\right.}} \sqrt{\frac{\mu}{\rho t_{\text {oscill }}}}$,

where $t_{\text {oscill }}$ is a characteristic droplet oscillation time given by $t_{\text {oscill }}=\sqrt{\rho d^{3} / \sigma}$. Using a pre-factor $p=2.38$, the model was shown to give excellent agreement with experimental results for $\mathrm{Oh}<0.1$, and with the loss factor, $q$, adjusted to $q=0.025(1-a)$. The authors also showed that for $a \sim 0.65$, the model still shows a reasonable agreement with the experiments, when a pre-factor of $p=2.5$ and $q=0$ are used.

\section{Experimental methodology}

\subsection{The apparatus}

The experimental setup is illustrated in Fig. 3. The droplets are generated using custom-made continuous monodisperse nozzles. A $152 \mu \mathrm{m}$ ID nozzle is used in this study, which produces droplets size of 360-390 $\mu \mathrm{m}$. The dynamics of the collisions are captured using high-speed imaging techniques. The four regimes observed in the experiments: bouncing, fast coalescence, reflexive separation and 


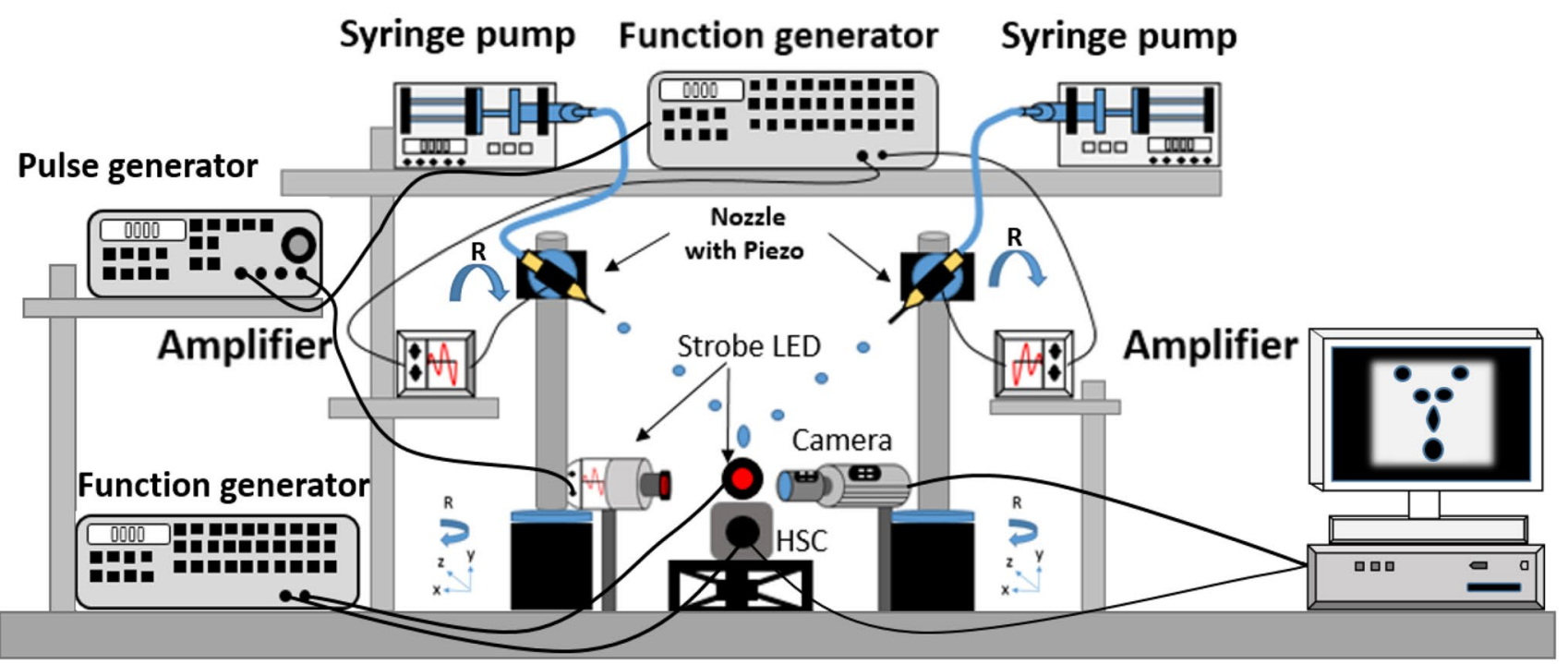

Fig. 3 Droplet collision rig, reproduced from (Al-Dirawi and Bayly 2019), with the permission of AIP Publishing

Fig. 4 Collision outcomes of $360 \mu \mathrm{m}, 2 \%$ HPMC droplets, $\mathrm{Oh}=0.021$

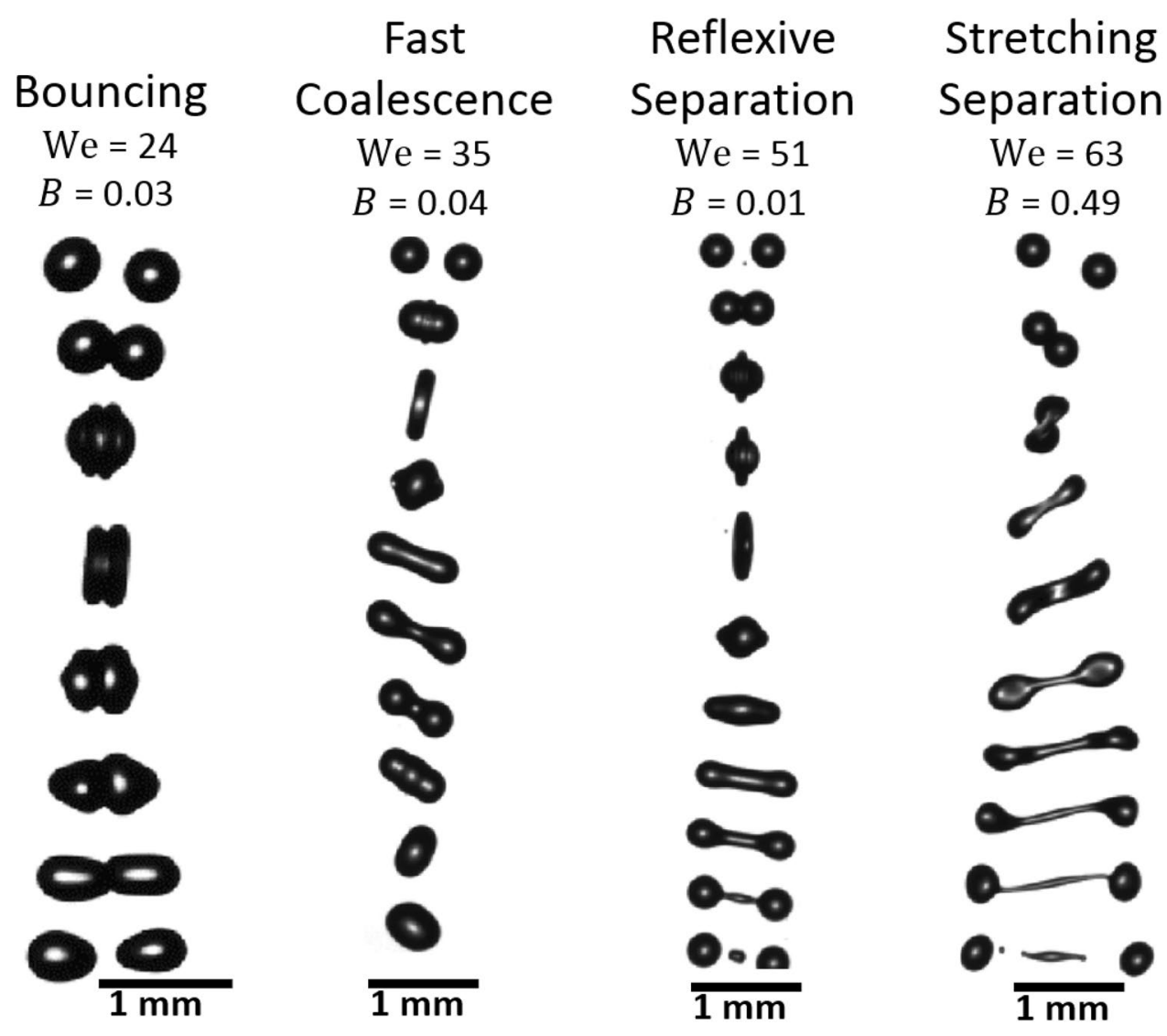

\subsection{Droplet fluids and experiments}

Three different concentrations, $2 \%, 4 \%$ and $8 \%$, of hydroxypropyl methylcellulose grade 603 Shin-Etsu Chemical's PHARMACOAT $^{\circledR}$ (HPMC) aqueous solutions were used. The rheology viscosity of the solutions was measured in a 
Table 1 Physical properties of the three HPMC systems that are used in this work at $20^{\circ} \mathrm{C}$

\begin{tabular}{lllcl}
\hline Type of liquid & $\rho\left(\mathrm{kg} \mathrm{m}^{-3}\right)$ & $\sigma\left(\mathrm{mN} \mathrm{m}^{-1}\right)$ & $\mu(\mathrm{mPa} \mathrm{s})$ & Oh \\
\hline 2\% HPMC & 1000 & 46.00 & 2.80 & 0.021 \\
$4 \%$ HPMC & 1000 & 45.80 & 8.20 & 0.063 \\
$8 \%$ HPMC & 1000 & 45.72 & 28.40 & 0.216 \\
\hline
\end{tabular}

rheometer (Anton Paar, Physica MCR 301) using a cone and plate geometry ( $75 \mathrm{~mm}$, Angle $1^{\circ}$, and gap $0.149 \mathrm{~mm}$ ) and a linear shear sweep from 1 to $1000 \mathrm{~s}^{-1}$ over $410 \mathrm{~s}$ at $20^{\circ} \mathrm{C}$. $2 \%$ HPMC and 4\% HPMC solutions showed Newtonian behaviour and a constant viscosity over the shear rate range tested. On the other hand 8\% HPMC showed a narrow shearthinning range at the shear rate from 0 to $100 \mathrm{~s}^{-1}$ at which the viscosity decreases by $10 \%$ then behaved as Newtonian fluid from 100 to $1000 \mathrm{~s}^{-1}$. All solutions' physical properties, i.e., viscosity, surface tension and density, are detailed in Table 1. More details on the methods of measurements in our previous work Al-Dirawi and Bayly (2019).

In our previous work (Al-Dirawi and Bayly 2019), regime maps, for collisions of identical droplets, were generated using the three solutions 2\% HPMC, $4 \%$ HPMC and $8 \%$ HPMC. In this study, the same solutions were used but to generate regime maps for collisions of droplets with nonidentical viscosities.

In addition to these experiments, some coloured images were obtained to help in understanding the dynamics of the collisions. This was done by adding $300 \mathrm{ppm}$ of Nigrosin water-soluble dye to the higher viscosity droplet to make it black while keeping the low viscosity droplet transparent. To distinguish between the colours of the two droplets, the droplets were lit using a front light and a white background was used. The light was positioned at an angle of $\sim 45^{\circ}$ compared to the high-speed camera to obtain a good colour contrast.

\subsection{Tracking methodology}

To extract the impact details of the droplets from the high frame rate videos, a customised tracking algorithm was used based on a Matlab code combined with the DMV code by Basu (2013). This algorithm is explained in detail in our previous work, Al-Dirawi and Bayly (2019).

\section{Results}

\subsection{Regime maps}

The standard regime maps of droplet collisions, with droplets that have identical physical properties, are commonly plotted in the parameter space of We and $B$. However, for collisions of droplets with different physical properties, We is not unique as it can have different values depending on the physical properties chosen. Gao et al. (2005) suggested that in the case of droplets of two different miscible liquids, We should be based on the properties of the droplet that has lower surface tension. This was attributed to the belief that the lower surface tension controls whether the collision outcome is coalescence or separation. However, this is only valid for collisions of low viscosity droplets because of the predominance of the viscosity effect in determining the collision outcome in viscous collisions (Kuschel and Sommerfeld 2013). Therefore, in some studies of collisions with non-identical fluids, the use of We in the regime maps is avoided and the relative velocity is used instead, such as in Planchette et al. (2010).

In this work, HPMC solutions show a negligible variation in surface tension and density, as seen in Table 1. We is, therefore, independent of solution concentration and allows the regime maps to be constructed based on We. Moreover, the similar values of surface tension and the density, see Table 1, isolate the effect of the viscosity on the collision outcome.

To allow comparison and further analysis, the three regime maps reported in our previous work, Al-Dirawi and Bayly (2019), for collisions of identical droplets for the three solutions, 2\%, 4\%, and 8\% HPMC, are shown in Fig. 5. In this work, this data was extended, to complete the matrix of the six possible combinations of the three HPMC solutions, by constructing three more regime maps for collisions of droplets with non-identical viscosities, as shown in Fig. 6. Noticeably, the regime maps of the non-identical solutions show defined regime boundaries that are qualitatively comparable to those of identical solutions.

In the following sections, the effect of the viscosity on the regime maps and the dynamics of the collisions will be discussed by comparing the role of the viscosity on collisions of droplets with identical viscosities with its role on non-identical collisions. Finally, the applicability of the existing models of the regimes boundaries, on both types of collisions (i.e., of identical and non-identical viscosities), will be discussed.

\subsection{Comparison of the identical and the non-identical collisions}

\subsubsection{Bouncing}

Although the role of the viscosity on collisions of identical droplets has been widely investigated, its effect on the bouncing regime boundary has scarcely been studied. This might be due to the limited number of studies that show a clear transition from the bouncing regime to the fast 

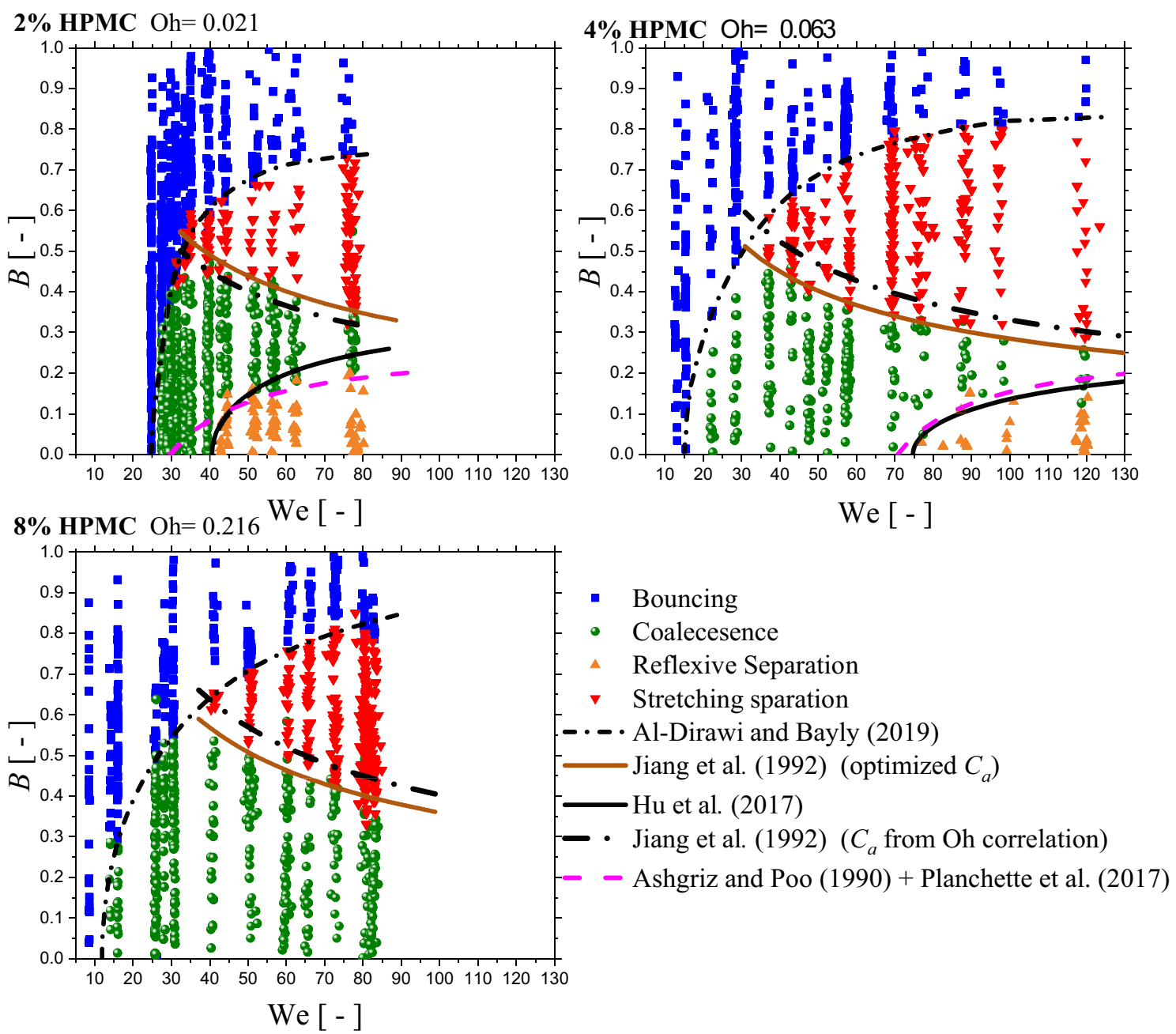

Fig. 5 Regime maps of binary droplet collisions with droplets that have identical viscosities, and the performance of the existing models in predicting the regimes' boundaries Adapted from Al-Dirawi and Bayly (2019), with the permission of AIP Publishing

coalescence regime at head-on collisions $\left(\mathrm{We}_{\mathrm{B} / \mathrm{FC}}\right)$. The absence of a bouncing to fast coalescence transition at head-on collisions could be attributed to the droplets' fluid properties, as some fluids do not show bouncing at low $B$, such as water (Qian and Law 1997) and ethanol (Estrade et al. 1999), or they do not show any bouncing at the entire range of $B$, such as milk (Finotello et al. 2018b). Krishnan and Loth (2015) suggested based on a literature survey that for head-on collisions, $\mathrm{We}_{\mathrm{B} / \mathrm{FC}}$ value would be expected to increase with increasing viscosity. However, the basis of this claim is based on extrapolation of data from high $B$ to low $B$, for fluids which are not seen to bounce at $B=0$, rather than direct collision data. Pan et al. (2016) also showed that a $30 \%$ aqueous glycerol solution, with a viscosity $3 X$ that of pure water, promotes bouncing compared to pure water droplets. The authors attributed this to less surface mobility in the case of higher viscosity droplets that suppresses the air drainage and hence bouncing is promoted.
The above hypothesis of surface mobility by Pan et al. (2016) is based on the observed phenomenon that when the droplets' interfaces approach each other the trapped air experiences high-pressure build-up at the centre of the gap, which causes indentation to the interfaces. Because of this indentation, a rim is formed on the surface and hence the minimum clearance between the droplets takes a ring shape. Therefore, to discharge the trapped air, this rim is pushed away from its centre. This dynamic is called surface mobility. Therefore, at higher viscosity the rim will have less mobility which resists the air drainage and consequently bouncing occurs. It should be noted that the surface mobility was numerically and experimentally observed in droplet coalescence on surfaces (Hicks and Purvis 2010; Kolinski et al. 2012), while in binary droplet collisions it is only observed in numerical studies (Pan et al. 2008, 2016).

In this study, the regime maps of all viscosity combinations show a bouncing to fast coalescence transition at 

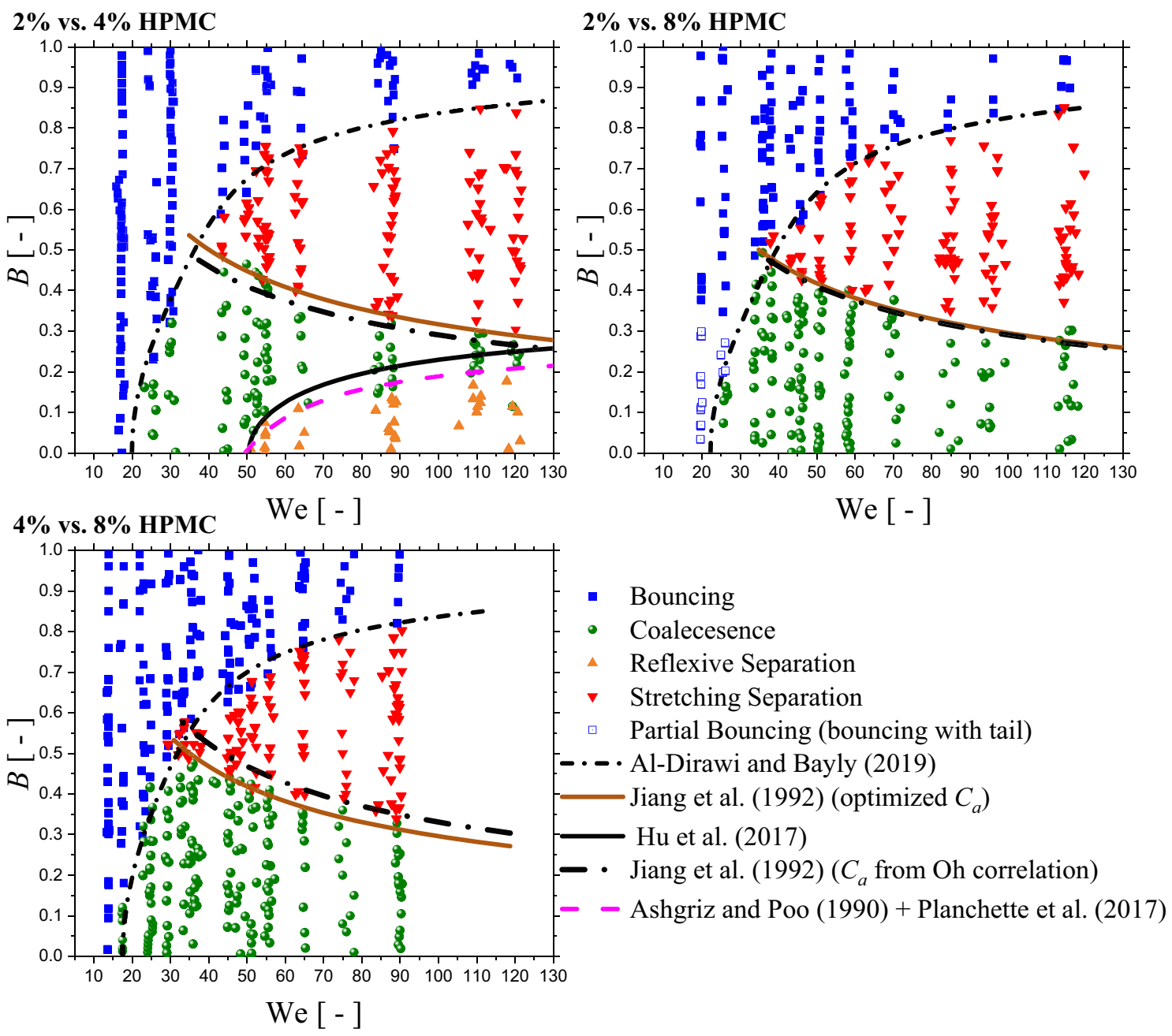

Fig. 6 Regime maps of binary droplet collisions with droplets that have non-identical viscosities, and the performance of the existing models in predicting the regimes' boundaries

head-on collisions. For collisions between droplets of identical fluid, $\mathrm{We}_{\mathrm{B} / \mathrm{FC}}$ decreases with increasing viscosity, as shown in Fig. 5 (Al-Dirawi and Bayly 2019). This is contrary to the aforementioned observations of Krishnan and Loth (2015) and Pan et al. (2016). This might be due to the higher viscosity range in this work compared to those in the previous studies, which are up to $4.3 \mathrm{mPa}$ s in Krishnan and Loth (2015) and up to $2.8 \mathrm{mPa}$ s in Pan et al. (2016). At high viscosity, the pressure build-up of the trapped air might not be sufficient to cause a significant surface indentation (Langley et al. 2017) and hence bouncing might be controlled by a different mechanism. By accepting this hypothesis, the resistance of the gas drainage is, therefore, determined by the area of surface flattening, which is expected to decrease by increasing the viscosity, as shown in the schematic in Fig. 7. Therefore, the bouncing might be enhanced by increasing the viscosity, according to the mechanism of the surface mobility, up to a certain limit after which the flattening mechanism takes over and hence increasing the viscosity further suppresses the bouncing. This can be a possible explanation for the suppression of bouncing when the droplet viscosity is increased.

Figure 8 shows the bouncing dynamics of the three HPMC systems. The viscosity of $2 \%$ HPMC is within the range reported by Krishnan and Loth (2015) and Pan et al. (2016); therefore, it is expected to have some surface indentation. This also can be expected by noticing the distorted shape of the droplet, at time $0.17 \mathrm{~ms}$ and $0.27 \mathrm{~ms}$ in Fig. 8, which seems to have a rim development. However, in cases of $4 \%$ and $8 \%$ HPMC the droplets do not show clear distortions and seem to have nearly flat interfaces (or negligible indentation). Thus, in 2\% HPMC we assume there is a surface mobility mechanism that controls the air drainage, whereas it is controlled by the flatting mechanism in $4 \%$ and $8 \%$ HPMC. However, more experimental and numerical efforts are required to validate the hypotheses of the 


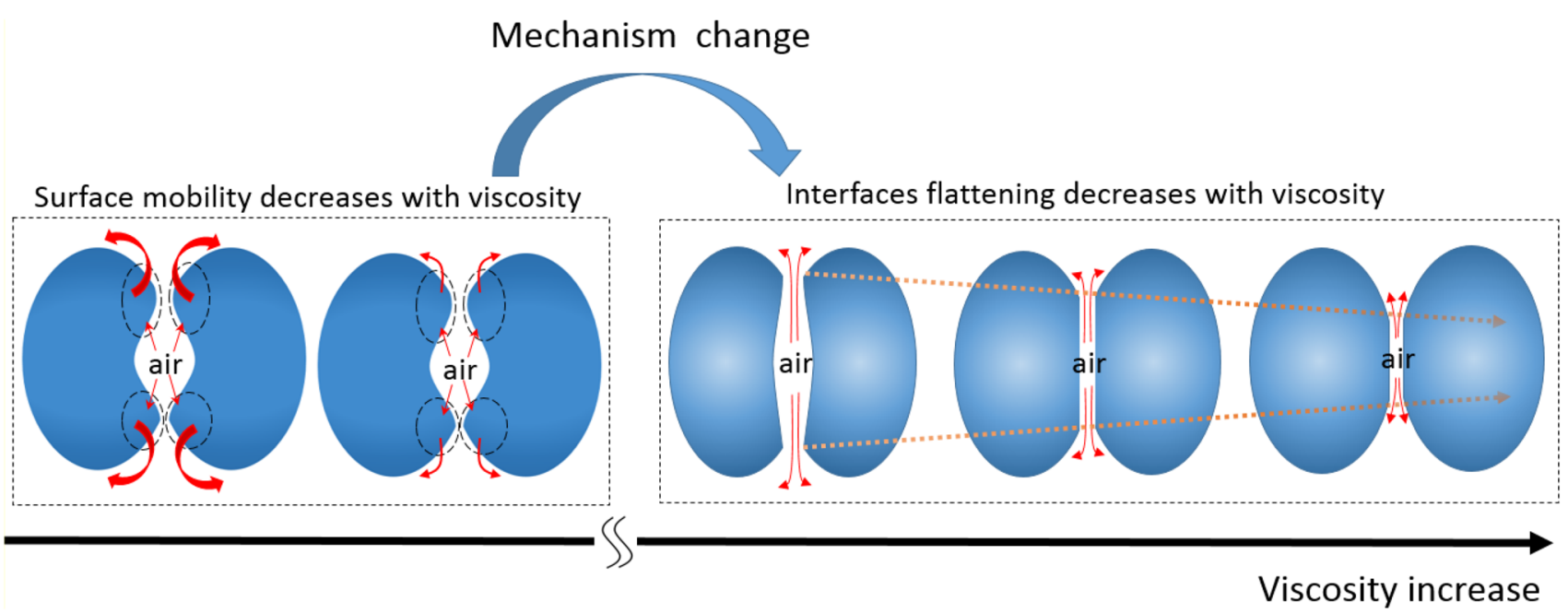

Fig. 7 Schematic showing the expected change in the mechanism of interface deformation and air drainage process on increasing droplet viscosity

Fig. 8 Effect of the viscosity on the dynamics of the head-on bouncing collision, at the transitional Weber numbers between bouncing and coalescence $\mathrm{We}_{\mathrm{B} / \mathrm{FC}}$, for droplets that have identical viscosities. The higher droplets viscosity the lower $\mathrm{We}_{\mathrm{B} / \mathrm{FC}}$ and deformation

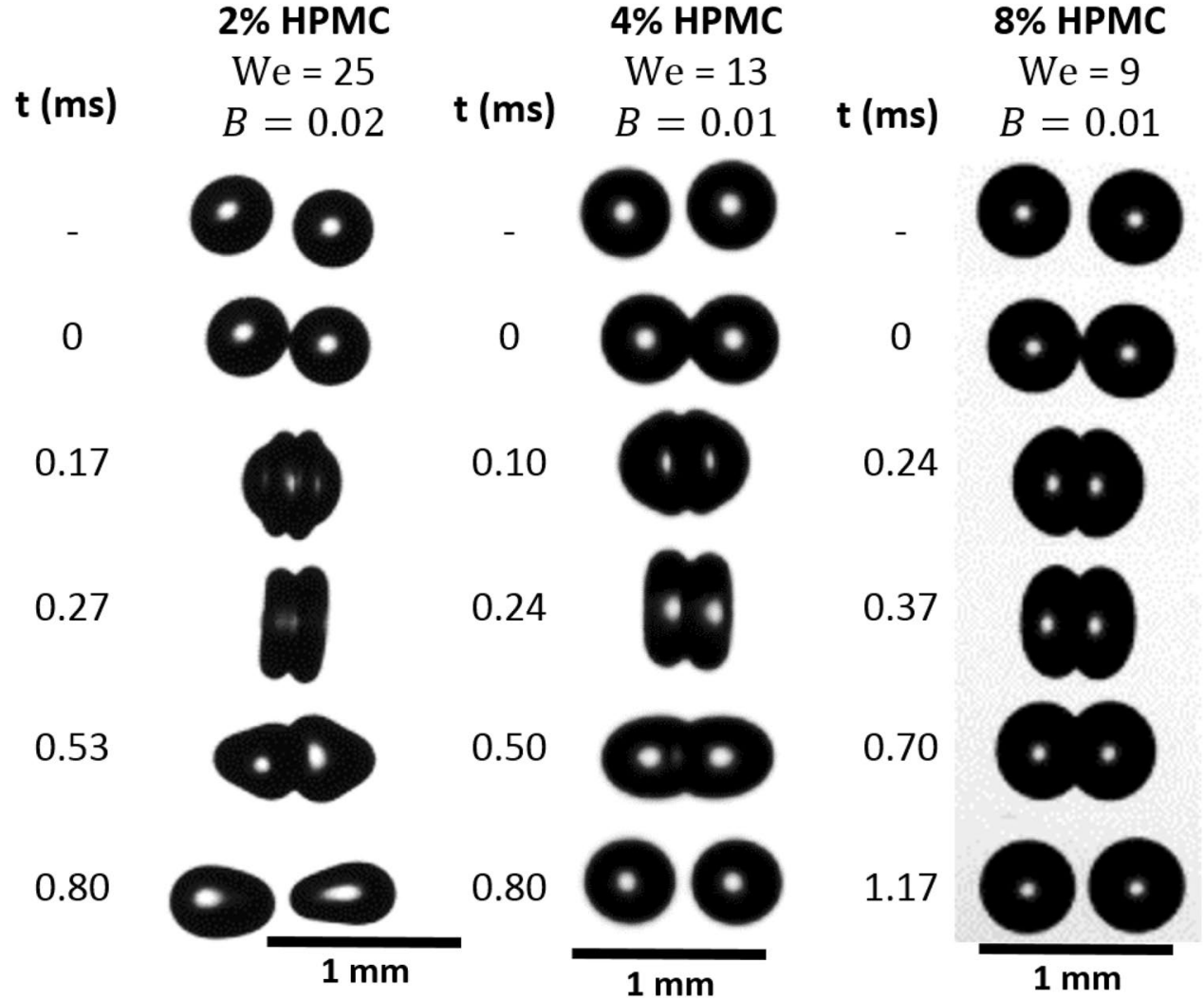

Table 2 Bouncing/fast coalescence boundary for head-on droplet collisions

\begin{tabular}{c|ccccc}
$\%$ HPMC & $2 \%$ & 2 vs. $4 \%$ & $4 \%$ & 4 vs. $8 \%$ & $8 \%$ \\
\hline$W e_{B / F C}$ & $26 \pm 1$ & $22 \pm 2$ & $18 \pm 2$ & $15 \pm 1$ & $11 \pm 2$ \\
$\%$ HPMC & & 2 vs. $8 \%$ & \\
\hline$W e_{B / F C}$ & \multicolumn{5}{c}{$21 \pm 2$}
\end{tabular}


Fig. 9 Effect of the viscosity on the dynamics of the head-on bouncing collisions of droplets that have different viscosities. High viscosity (right droplet) low viscosity (left droplet) Partial bouncing is seen at high viscosity ratio ( $2 \%$ vs. $8 \%$ HPMC)
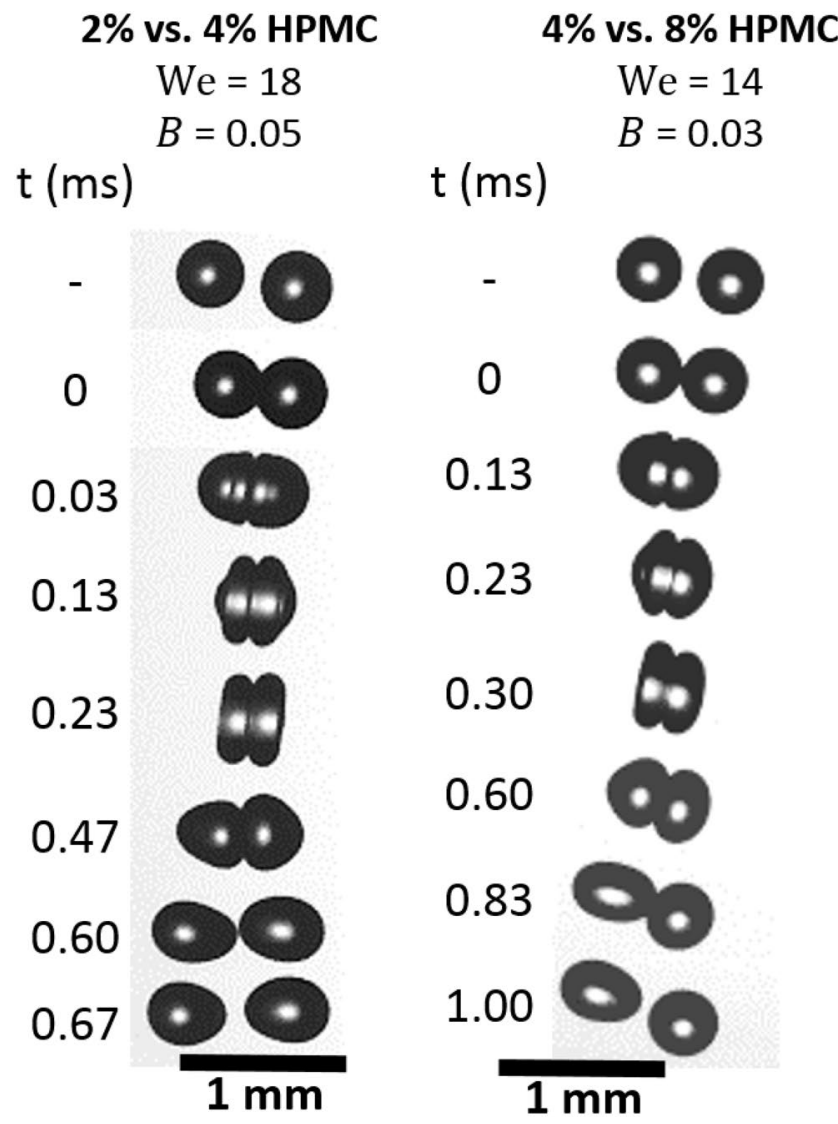

$2 \%$ vs. $8 \%$ HPMC

$\mathrm{We}=20$ $B=0.09$
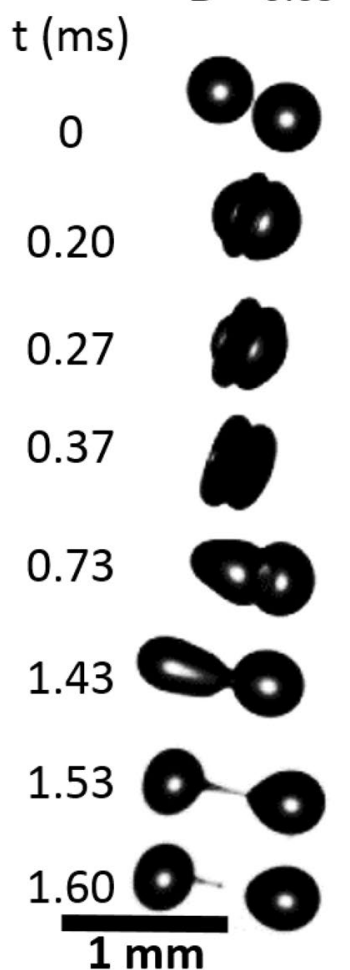

mechanism change with sufficient increase in the viscosity of the droplets.

In the case of collisions between miscible droplets that have different viscosities, $\mathrm{We}_{\mathrm{B} / \mathrm{FC}}$ has an intermediate value between the values of the identical cases, see Table 2 . The bouncing dynamic in cases of head-on identical collisions is symmetric to the impact plane, as shown in Fig. 8. However, bouncing in cases of non-identical viscosities shows asymmetric dynamics as the lower viscosity droplet is deformed more than the higher viscosity droplet. This deformation difference increases as the viscosity ratio increases, as shown in Fig. 9. This change in deformation leads to a higher drainage rate of the trapped air between the colliding droplets than that of the equivalent case of the lower viscosity droplet, and a drainage rate lower than that of the equivalent case of higher viscosity droplets. Thus, the intermediate values of $\mathrm{We}_{\mathrm{B} / \mathrm{FC}}$ are expected.

A new collision regime has also been identified in case of non-identical viscosities at high viscosity ratio (i.e., $2 \%$ vs. $8 \%$ HPMC), where a relatively significant asymmetry in dynamics due to the differences in viscosity is observed. In this case, bouncing is accompanied by a temporary partial coalescence and a thin ligament (tail) between the two droplets is observed. This ligament breaks rapidly at the surface of the high viscosity droplet and retracts back to the lower

viscosity droplet, as shown in Fig. 9. After the pinch-off of the tail the droplets show no change in their original volume. This type of bouncing is named partial bouncing on the regime map in Fig. 6. The exact mechanisms that lead to the partial bouncing are not clear due to the complexities of surface deformation, air drainage and surface contact involved in determining the droplet dynamics in this region of the regime map.

It is interesting to notice that the dynamics of bouncing in case of non-identical viscosities is very similar to the bouncing dynamics of the collisions of low viscosity droplets that have large surface tension difference, such as the bouncing collision between a water droplet and a diesel oil droplet, see Fig. 9 in Chen and Chen (2006). In this case, the droplet with lower surface tension, diesel oil, suffers larger deformation than that of the water droplet. This is because the higher surface tension leads to higher capillary pressure $(2 \sigma / d)$, which makes the droplet more resistant to the deformation, and vice versa.

\subsubsection{Stretching separation}

Kuschel and Sommerfeld (2013) observed that increasing the viscosity in identical droplet collisions would shift the boundary of the stretching separation toward higher 

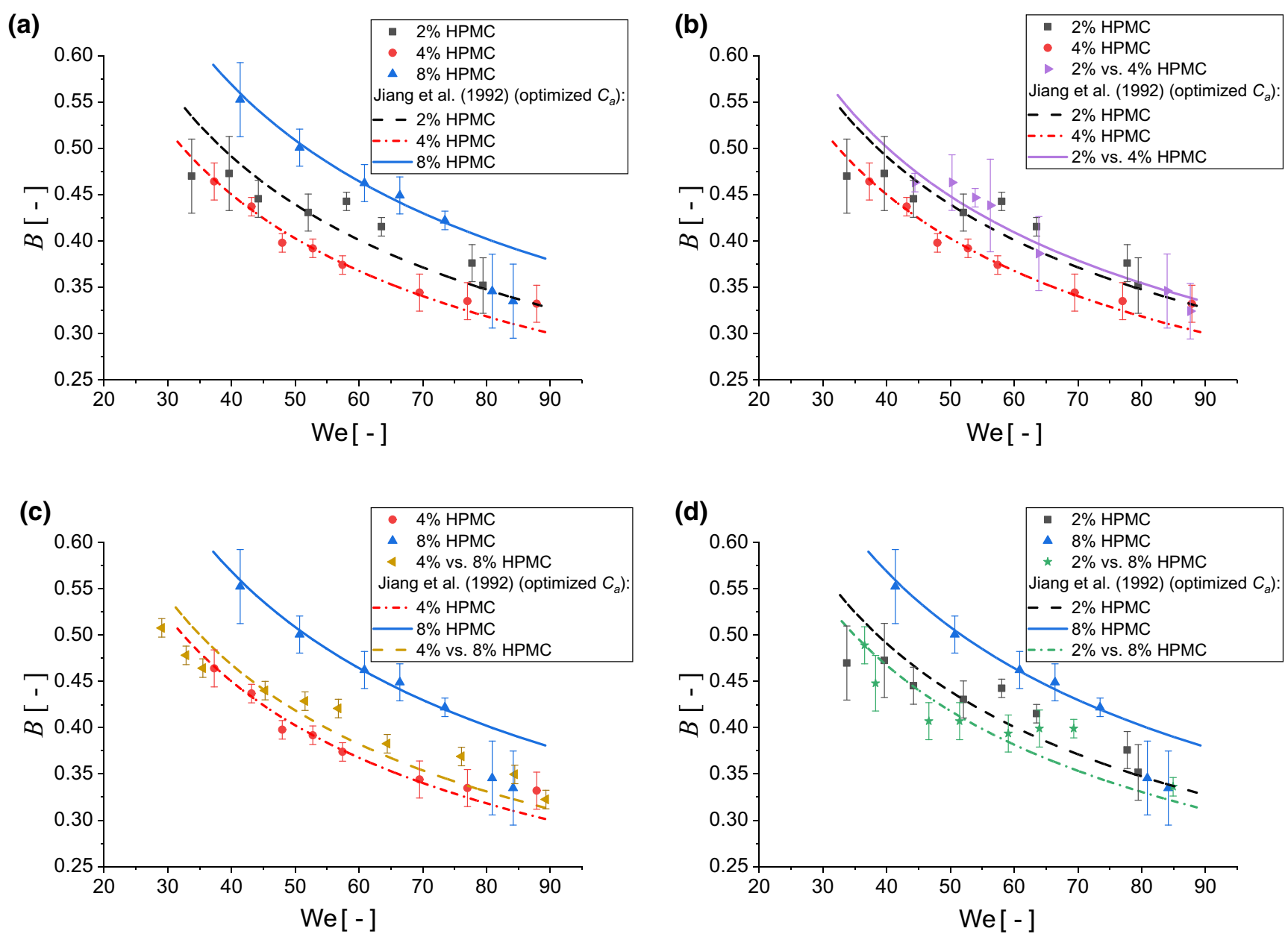

Fig. 10 Comparisons of the stretching separation boundaries of the identical droplets collisions at different viscosities, as well as the nonidentical droplets collisions vs. the identical droplets collisions. The

error bars represent the uncertainty due to the gaps between the data points in Figs. 5 and 6 . The lines represent the model of Jiang et al. (1992) with $C_{b}=1$ and $C_{a}$ is optimised based on the minimum MAE

$B$ values. The regime maps of the identical cases in this study show the same trend, when HPMC concentration is increased from $4 \%$ to $8 \%$, as shown in Fig. 10a. However, the boundary of $2 \%$ shows slightly higher $B$ values compared to 4\% HPMC, see Fig. 10a. This trend (the boundary of the lower viscosity collisions occurring at higher $B$ ) was also observed in Kuschel and Sommerfeld (2013) in case of comparing the regime maps of $20 \%$ and $40 \%$ saccharose. A possible hypothesis explaining this will be given in the following discussion.

In the three non-identical systems studied, the boundary of the stretching separation regime is nearly superimposed (with slightly higher B) on the boundaries of the identical viscosity cases of the lower viscosity droplet, as shown in Fig. 10b-d. This can be clearly seen in case of $4 \%$ vs. $8 \%$ HPMC, where its boundary closer to the identical case of $4 \%$ HPMC than the identical case of $8 \%$ HPMC, as shown in Fig. 10c. Similar behaviours can be seen in cases of $2 \%$ vs. $4 \% \mathrm{HPMC}$ and $2 \%$ vs. $8 \%$ HPMC as their stretching

separation boundaries are closer to the cases of the identical viscosity collisions of the lower viscosity (i.e., 2\% HPMC in both cases), as shown in Fig. 10b, d. Ultimately, this means increasing the viscosity of one of the colliding droplets has a negligible effect on the boundary of the stretching separation regime.

It is interesting to note that both $2 \%$ and $2 \%$ vs. $4 \%$ HPMC boundaries lie at higher $B$ values than 4\% HPMC, see Fig. 10b, which is contrary to the other aforementioned cases. This may be due to the dominance of surface tension, $\mathrm{Oh}<0.1$, in controlling collision outcome in these conditions. A slight increase in the viscosity (from $2.8 \mathrm{mPa}$ in $2 \% \mathrm{HPMC}$ to $8.2 \mathrm{mPa} \mathrm{s}$ in $4 \%$ HPMC) might reduce this effect before entering an area, where viscosity plays a dominant role in governing behaviour. In Fig. 10a, 8\% HPMC the boundary occurs at higher $B$ than the lower viscosity cases, $\mathrm{Oh}=0.216$, which suggests that viscosity will have a dominant effect. It is interesting to note that Planchette et al. (2017) also reported similar Oh boundary behaviour 
Fig. 11 Shadow images show the effect of the viscosity on the dynamics of the stretching separation collisions of droplets that have non-identical viscosities. The high viscosity droplet coming from the right (on the top) and stretching to the left (from the middle to the bottom of the images)

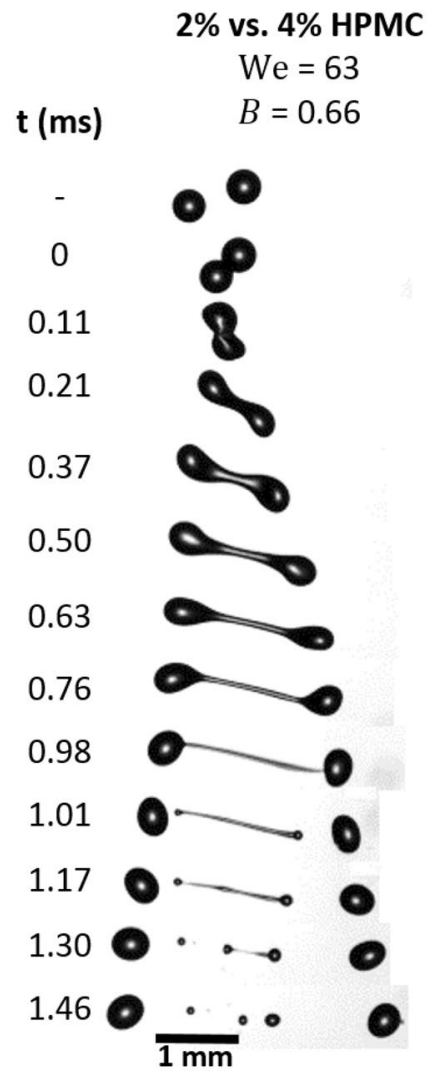

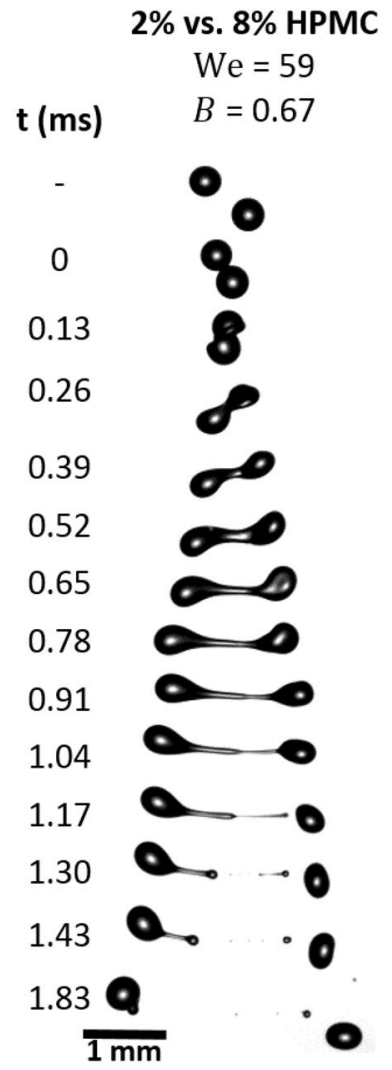

$4 \%$ vs. $8 \%$ HPMC $\mathrm{We}=64$ $B=0.65$

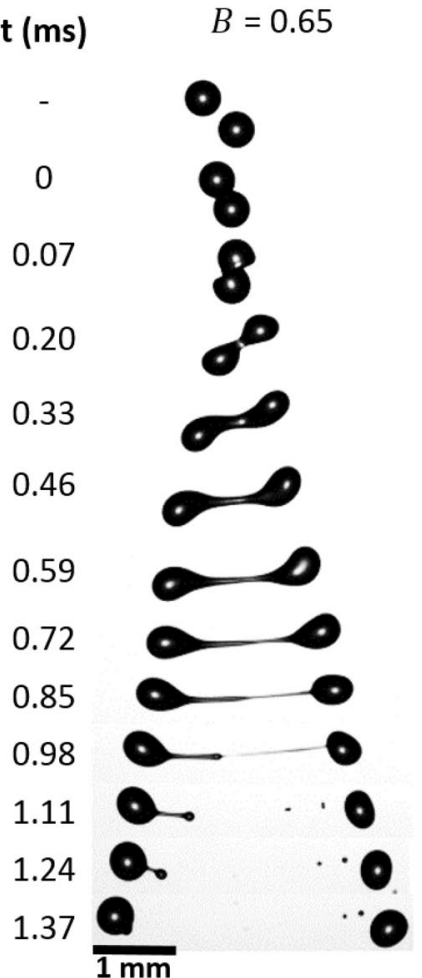

for head-on collisions, as at $\mathrm{Oh}<0.1$ the compression period shows different deformation and has a lower viscous loss ratio $a$, which is attributed to insufficient time, at low Oh, to form viscous boundary layers. However, a full understanding of these observations requires a systematic study of the viscosity effects for solutions that have $\mathrm{Oh}<0.1$, which is out of the scope of this research.

In cases of collisions between droplets that have identical viscosities, a uniform ligament is formed between the droplets during the stretching process, as shown in Fig. 4. However, in case of non-identical viscosities, the ligament is thicker on the side of the higher viscosity droplet and the break-up happens at the thinner side of the ligament, near the low viscosity droplet, as shown in Fig. 11. Consequently, it is of interest to visualise and understand the mixing between the two droplets that occurs during the stretching separation process. As this is not possible from the shadow images in Fig. 11 an alternative imaging setup was developed, where one droplet was coloured and the collisions were lit from the front.

Colour images of stretching separation of the three viscosity ratios are shown in Fig. 12. During the early stages of the stretching process, a steep colour, and consequently viscosity, gradient is seen in the ligament near the higher viscosity droplet. This explains the non-uniform ligament in Fig. 11 and reveals that the break-up occurs in the lower viscosity region of the filament close to the low viscosity droplet. Thus, in these non-identical cases the stretching separation is controlled by viscosity of the lower viscosity droplet, which explains the near superposition of the stretching separation boundary of the non-identical droplets with the boundary seen for identical, lower viscosity droplets. Moreover, from Fig. 12, it also can be noticed that the separated droplets have no significant mixing, as they keep their original colour and no significant size change is observed, while the satellite droplets seem to have some mixing as their colour is in-between that of the original droplets.

For completeness, it should be noted that in collisions of immiscible droplets with different viscosities, Planchette et al. (2012) also found that the ligament is formed from the low viscosity droplet (the encapsulating droplet).

\subsubsection{Reflexive separation}

It is known from the previous studies of identical droplet collisions that increasing the viscosity shifts the onset of the reflexive separation $\mathrm{We}_{\mathrm{FC} / \mathrm{RS}}$ towards higher We (Kuschel and Sommerfeld 2013; Finotello et al. 2018a, b; Gotaas et al. 2007). The collisions of identical viscosity droplets show the same trend in this study. For example, changing the concentration of HPMC from $2 \%$ to $4 \%$ shifts $\mathrm{We}_{\mathrm{FC} / \mathrm{RS}}$ from $41 \pm 1$ 
Fig. 12 Coloured time-resolved images show that ligament is drawn from the low viscosity droplet. The transparent droplets are the lower viscosity, and the dark droplets are the higher viscosity
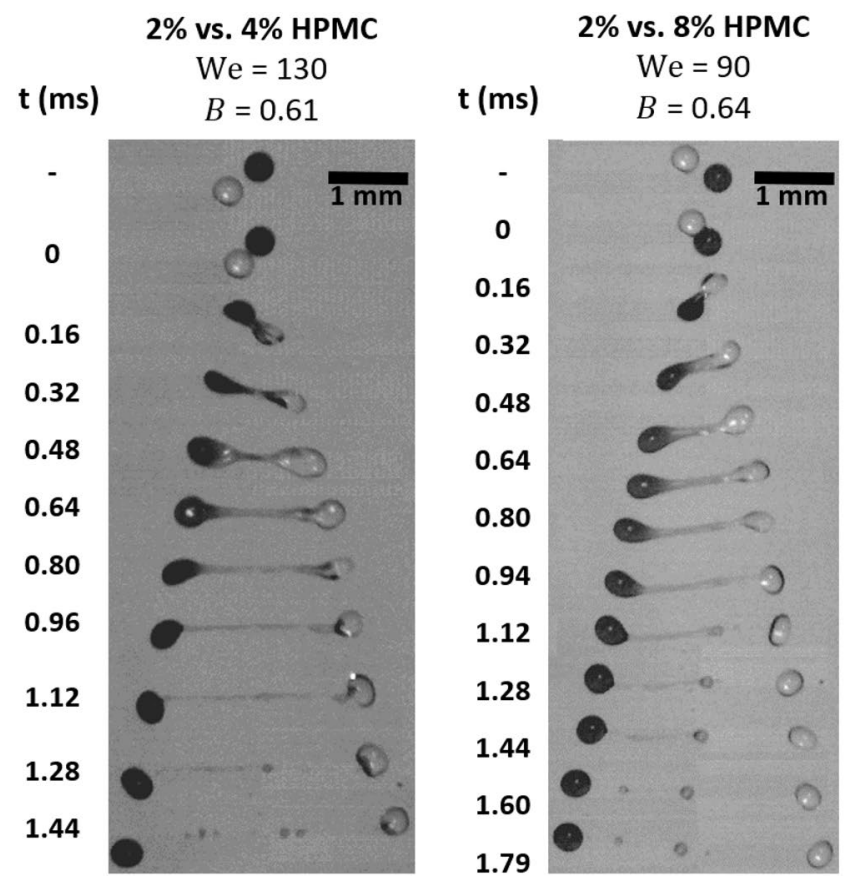

$2 \%$ vs. $8 \%$ HPMC

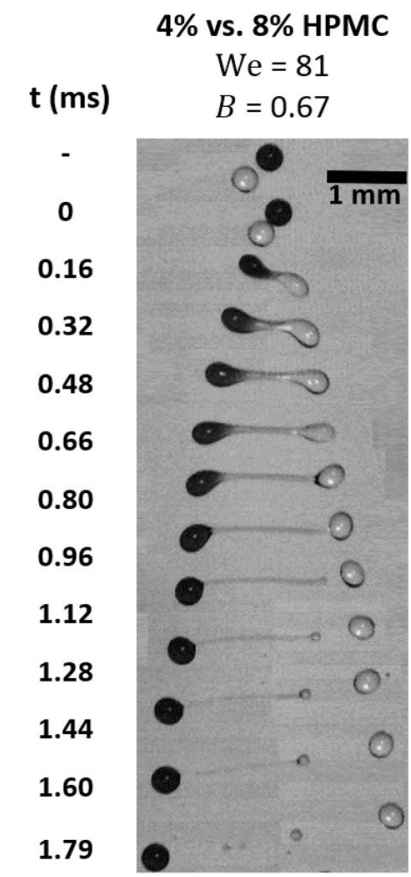

to $75 \pm 2$ and increasing the viscosity further in $8 \%$ HPMC shows no reflexive separation for the investigated range up to $\mathrm{We}_{\mathrm{Max}}=84$.

Unlike stretching separation, increasing the viscosity of one of the colliding droplets noticeably shifts the boundary of the reflexive separation towards higher We. However, this shift is less significant than that of increasing the viscosity of both of the colliding droplets. For example, $\mathrm{We}_{\mathrm{FC} / \mathrm{RS}}$ of the collision of $2 \%$ HPMC droplet with 4\% HPMC droplet is $48 \pm 2$, while it is $41 \pm 1$ and $75 \pm 2$ for identical viscosity collisions of $2 \%$ and $4 \%$, respectively, see Figs. 5 and 6. Interestingly, on further increasing the viscosity of the high viscosity droplet, $2 \%$ vs. $8 \%$ HPMC, no reflexive separation is observed within the investigated range of We. Similarly, in $4 \%$ vs. $8 \%$ HPMC, no reflexive separation is observed, see Fig. 6.

In the case of head-on collisions of identical droplets, the droplets initially form a rimmed lamellar disc, whereby the impact kinetic energy is completely converted into viscous loss and surface energy. Then, by the action of surface tension, the edges of the formed disc retract toward the centre of the mass. This retraction causes a reflexive internal flow inside the combined droplet which leads to the formation of a cylindrical shape aligned along the original axis of collision. At high enough We, this continues to extend symmetrically until separation occurs. When the impact We is relatively low i.e., at $\mathrm{We}_{\mathrm{C} / \mathrm{RS}}$, the separation leads to formation of two equal-size droplets that have equivalent size to the mother droplets. However, at higher We a large satellite type droplet can form between the two droplets separating droplets. The size of this satellite droplet is proportional to
We (Ashgriz and Poo 1990). Figure 13 shows the dynamics of reflexive separation at relatively high We for $2 \%$ and $4 \%$ HPMC solutions.

In the case of non-identical viscosity collisions a similar rimmed lamellar disc is still observed; however, on retraction the internal reflexive flow shows non-symmetrical behaviour. In these cases, the lower viscosity fluid starts separating faster and forms a baseball-bat shape. The subsequent ligament breaks near the end of the low viscosity region forming a droplet. The remaining extended ligament then retracts towards the high viscosity end forming a single droplet with a larger size than the lower viscosity droplet. The coloured droplet images help to understand this behaviour. Interestingly, some partial mixing is seen between the colliding droplets in the earlier stages of the collision. This leads to a viscosity gradient along the ligament and, consequently the break-up occurs at the lower viscosity end of the ligament, closest to the low viscosity droplet. At the collision conditions shown in the last set of images in Fig. 13, the remaining ligament retracts and forms a single large droplet. This large droplet will be more diluted than the initial droplet due to the partial mixing of the retracted ligament; however, the small droplet has a concentration similar to that of the lower viscosity.

\subsection{Boundaries Modelling}

Modelling the regime boundaries of binary droplet collisions has received substantial attention. However, the majority of these studies deal with collisions of droplets that have identical fluids (e.g., Ashgriz and Poo 1990; Jiang et al. 1992; 
Fig. 13 Dynamics of reflexive separation collisions for droplets that have identical and non-identical viscosities. In the case of the collision of $2 \%$ vs. $4 \%$ HPMC in the shadow images, the low viscosity droplet is located on the left). In the coloured images, the dark droplet is the higher viscosity (4\% HPMC)

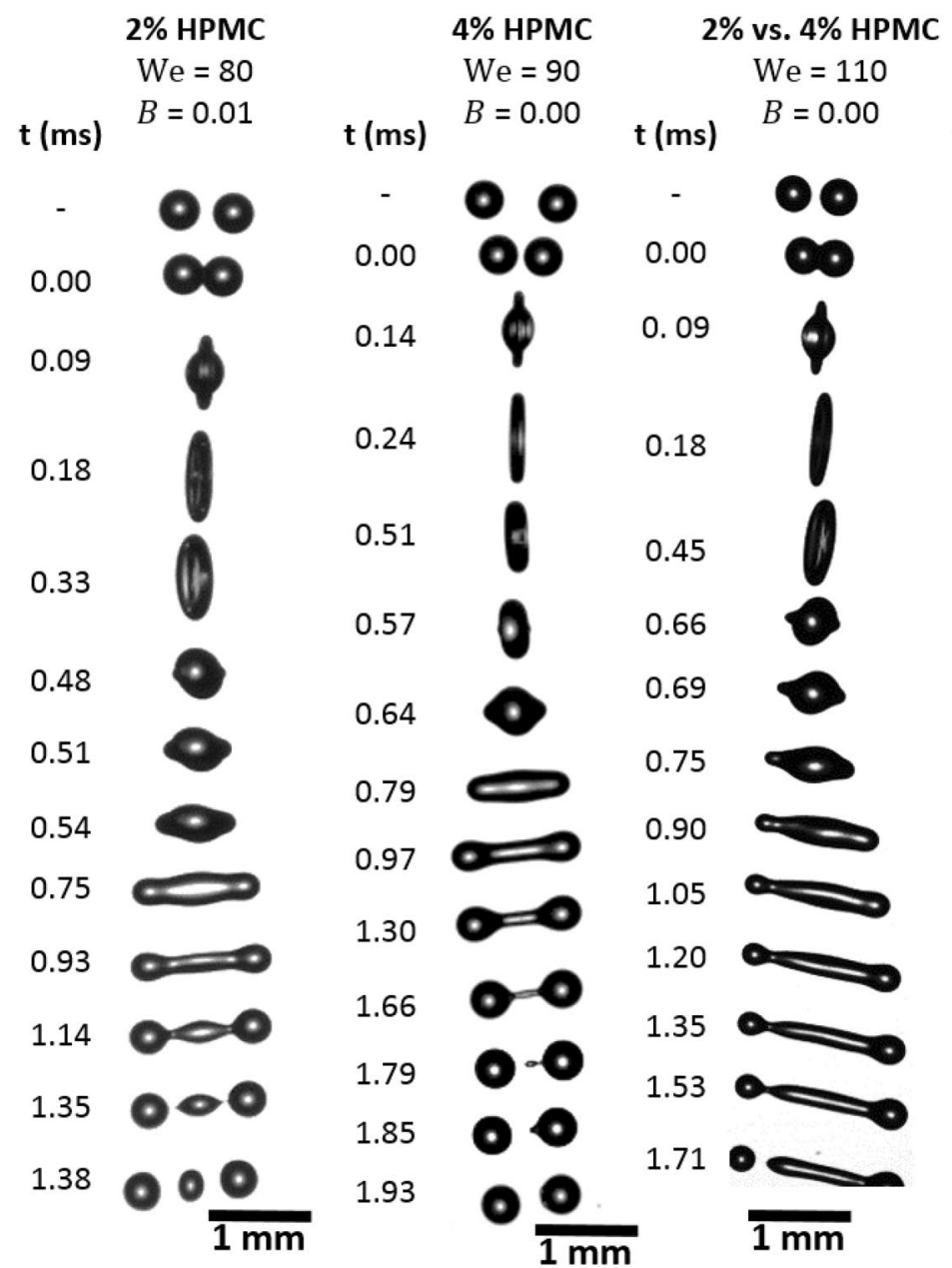

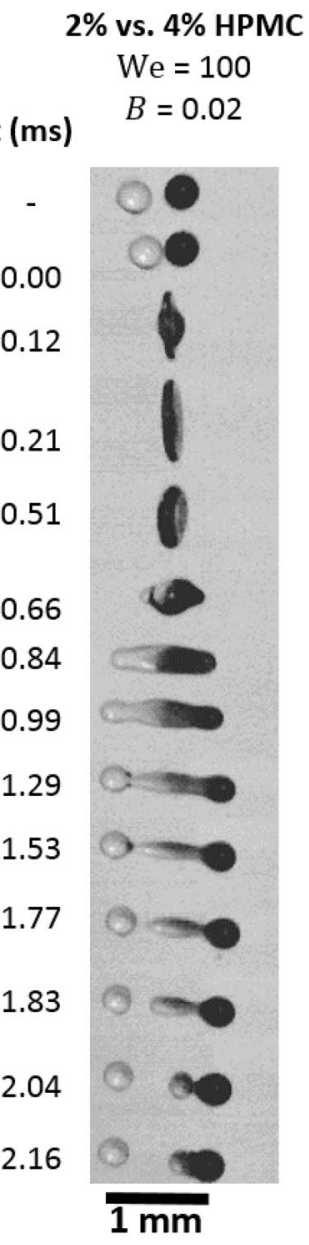

Estrade et al. 1999). In addition, a few studies have been conducted to model the boundaries of the separation regimes in collisions of immiscible droplets (Planchette et al. 2012). To the best knowledge of the authors, the regime boundaries of non-identical, miscible fluid, droplet collisions have not been considered in the literature. In this section, the applicability of the existing models (see Sect. 2) for the boundaries of the regimes for both identical and non-identical viscosity droplet collisions, of this study, will be examined.

\subsubsection{Bouncing boundary}

The bouncing boundary was fitted using the model of AlDirawi and Bayly (2019). Initially, the shape factor was evaluated at $B=0$ using Eq. (4), which is a function of $e_{1}$ and $e_{\mathrm{s}}$. It should be noted that, $e_{l}$ and $e_{s}$ are identical in case of collisions of identical droplets, while they are different in case of collisions of droplets with non-identical viscosities. At head-on collisions, $e=\left(1-c^{2} / a^{2}\right)^{0.5}$, thereby Eq. (4) is a function of $c_{1}, c_{\mathrm{s}}, a_{1}$ and $a_{\mathrm{s}}$, which were measured directly from the images at $\mathrm{We}_{\mathrm{B} / \mathrm{FC}}$ using ImageJ. Equation (3) then was used to estimate $\mathrm{We}_{\mathrm{B} / \mathrm{FC}}$ at $B=0$. The viscous loss factor $\alpha$ was estimated by fitting the model to the experimental data at $B=0$. This $\alpha$ value is then kept for the whole range of $B$. At $B>0, e=f(\Psi, \beta)$, the constants $\Psi$ and $\beta$ were optimised using the minimum mean absolute error (MAE) as in our previous work, Al-Dirawi and Bayly (2019). Table 2 summarises the values of the constants that are used in this model for different cases. The model shows excellent performance on the non-identical viscosities collisions, as shown in Fig. 6. It should be noted that the use of the model in the identical collisions cases, in Fig. 5, is that of our previous work (Al-Dirawi and Bayly 2019).

As it would be expected, the viscous loss $\alpha$ increases as the viscosity increases, which shown in Table 3. In the nonidentical viscosity cases, an intermediate $\alpha$ value is seen, which is consistent with the behaviour being an average of the two viscosities. On the other hand, the shape factor $\phi_{\mathrm{o} . \mathrm{s} .}^{\prime \prime}$ increases as the viscosity decreases; this is due to the increased deformation observed for low viscosity droplets (Al-Dirawi and Bayly 2019). In collisions of droplets with non-identical viscosities the values of the $\phi_{\mathrm{o} . \mathrm{s} .}^{\prime \prime}$ are in-between 
those of the identical cases, as listed in Table 2. This is because the colliding droplets have different deformation extents that produce an intermediate total surface area compared to those of the identical cases of the low and the high viscosity droplets. The intermediate values of $\phi_{\mathrm{os}}^{\prime \prime}$ quantitatively explain the intermediate position of $\mathrm{We}_{\mathrm{B} / \mathrm{FC}}$ in the non-identical case compare to the identical cases.

It should be noted that, in non-identical viscosity collisions, $\alpha$ and $\phi_{\mathrm{o} . \mathrm{S}}^{\prime \prime}$ are not an exact average of the values of the two identical cases of the low and the high viscosity systems. This is because the transitional points from bouncing to fast coalescence $\left(\mathrm{We}_{\mathrm{B} / \mathrm{FC}}\right)$ are not the average of those of the identical cases. This might be attributed to the complexity of the dynamics related to the behaviour of the trapped air film between the droplets.

\subsubsection{Stretching separation boundary}

The boundary of the stretching separation regime, in case of non-identical viscosities, is comparable to that of the identical case of the lower viscosity droplet. Therefore, the model of Jiang et al. (1992), Eq. (10), can be used to fit the boundaries in both identical and non-identical collisions. In the case of collisions of droplets with non-identical viscosities, the lower viscosity is used in the model. This is based on our aforementioned observation that the ligament between the droplets is mainly composed of the lower viscosity fluid and the break-up occurs near the lower viscosity droplet. Figures 5 and 6 show that the model of Jiang et al. (1992) captures the shape of the boundary very well for both the identical and non-identical cases. Also shown in these figures is a comparison of a fit with an optimised empirical parameter, $C_{a}$, with the fit using the $C_{a}=f(\mathrm{Oh})$ correlation of Sommerfeld and Pasternak (2019), Eq. (11). In both cases $C_{b}=1$, and the optimisation was done by minimising the MAE, using Eq. (18) and the data points, as shown in Fig. 10:

MAE $=\frac{1}{n} \sum_{i=1}^{n}\left|B_{\text {model }}-B_{\text {exp. }}\right|_{i}$.

Table 3 Bouncing model, Eq. (3), parameters

\begin{tabular}{llllll}
\hline & $\alpha$ & $\begin{array}{c}\phi_{\text {o.s. }}^{\prime \prime} \text { (at } \\
\text { Eq. }\end{array}$ & $\Psi$ & $\beta$ & $e^{2}$ \\
\hline $2 \%$ HPMC & 0.11 & 1.46 & 0.86 & 2.75 & 0.895 \\
$4 \%$ HPMC & 0.23 & 1.24 & 1.05 & 3.93 & 0.81 \\
$8 \%$ HPMC & 0.44 & 1.14 & 1.11 & 4.70 & 0.71 \\
$2 \%$ vs. 4\% HPMC & 0.14 & 1.36 & 1.32 & 4.21 & $0.88 ; 0.84$ \\
$2 \%$ vs. 8\% HPMC & 0.26 & 1.34 & 1.31 & 4.05 & $0.87 ; 0.84$ \\
$4 \%$ vs. 8\% HPMC & 0.3 & 1.25 & 1.15 & 3.76 & $0.79 ; 0.83$ \\
\hline
\end{tabular}

As can be seen in the case of the optimised empirical factor, the model of Jiang et al. (1992) gives a very good fit in all cases. However, the fit using the correlation in Eq. (11) (Oh for the lower viscosity droplet is used) gives a slight offset in most cases, though interestingly in the case of the non-identical collisions this offset in, $B$, is small $<\sim 0.05$.

Figure 14 details and quantitatively compares the values $C_{a}$ used to define the curves in these plots. Noticeably, the optimised $C_{a}$ values of the non-identical collisions are close to the values of the identical collisions of the droplet of the lower viscosity. Comparing optimised $C_{a}$ values with the $C_{a}$ values calculated using Eq. (11) a reasonable agreement is seen; however, there is a little scatter. Ultimately, the model of Jiang et al. (1992), with the single empirical parameter, $C_{a}$, can be used to accurately describe the stretching separation boundary for collisions of non-identical viscosities using the lower viscosity and $C_{b}=1$. The correlation of Sommerfeld and Pasternak (2019) provides a reasonable estimate for $C_{a}$, while an optimised value of $C_{a}$ gives an excellent fit of $B$ across a wide range of We.

The excellent agreement with the model of Jiang et al. (1992) for the boundary in all the systems studied, suggests that the impact parameter modifications proposed by Planchette et al. (2012), see Sect. 2.2, are not necessary, even though some droplet distortion and rotation was observed. However, it is also of interest to see if this alternative approach provides a good fit to the data, consequently, it was evaluated. The effective impact parameter $B_{\text {eff }}$, was calculated using the $8 \%$ HPMC boundary as a reference boundary based on the assumption that these droplets suffer negligible distortion compared to the lower viscosity cases. The empirical parameter $U^{*}$ was determined for both the identical and non-identical data sets by minimising the MAE between $B_{\text {eff }}$ describing each boundary and the reference boundary. Using these fitted values of $U^{*}$ to calculate $B_{\text {eff }}$ for the experimentally determined boundary values (Fig. 15a), shows a reasonable collapse on to a single curve, Fig. 15b, which can be approximated the model of Jiang et al. (1992). For completeness, as the model of Jiang et al. (1992) was fitted to this single curve, the empirical parameter $C_{\mathrm{a}}$ is dependent on the viscosity of the system, see Fig. 14. As all points collapsed on a single line, $C_{a}$ values of $2 \%$ and $2 \%$ vs. $4 \%$ and $2 \%$ vs. $8 \%$ HPMC were collapsed in a single value that is larger than the optimised values, similarly in case of $4 \% \mathrm{HPMC}$ and $4 \%$ vs. $8 \%$ HPMC.

The $U^{*}$ determined for each system are shown in the inset graph in Fig. 14. They correlate with the offsets in the boundaries shown in Fig. 10 which are discussed in Sect. 4.2.2. As the $8 \%$ HPMC case is significantly offset from the other curves, the values are relatively similar. These contrast with the findings of Planchette et al. (2012), who, for immiscible systems, reported higher absolute levels of $U^{*}$ and saw $U^{*}$ with increase monotonically with the viscosity of the more 


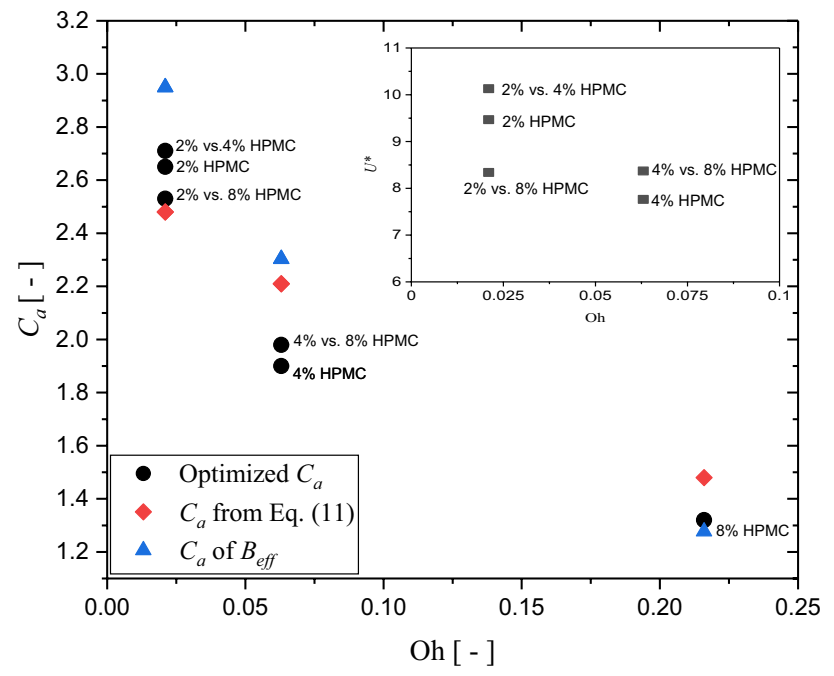

Fig. 14 Evaluation of the parameter $C_{a}$ in the modified model of Jiang et al. (1992), Eq. (10), for the six systems that are used in this study using different methods, where $C_{b}=1$. Circles are direct fittings based on the minimum MAE; diamonds are the values from the correlation of Sommerfeld and Pasternak (2019), Eq. (11); triangles are based on the effective impact parameter estimated from the model of Planchette et al. (2012). The inset figure shows the evaluation of the constant $U^{*}$ in the model of Planchette et al. (2012). Oh of the non-identical collisions is taken for the lower viscosity droplet

deformable component. Consequently, while this modelling approach can be used to approximate the boundary observed in these systems, the lack of clear trend in $U^{*}$ makes the approach of Jiang et al. (1992) preferable.

(a)

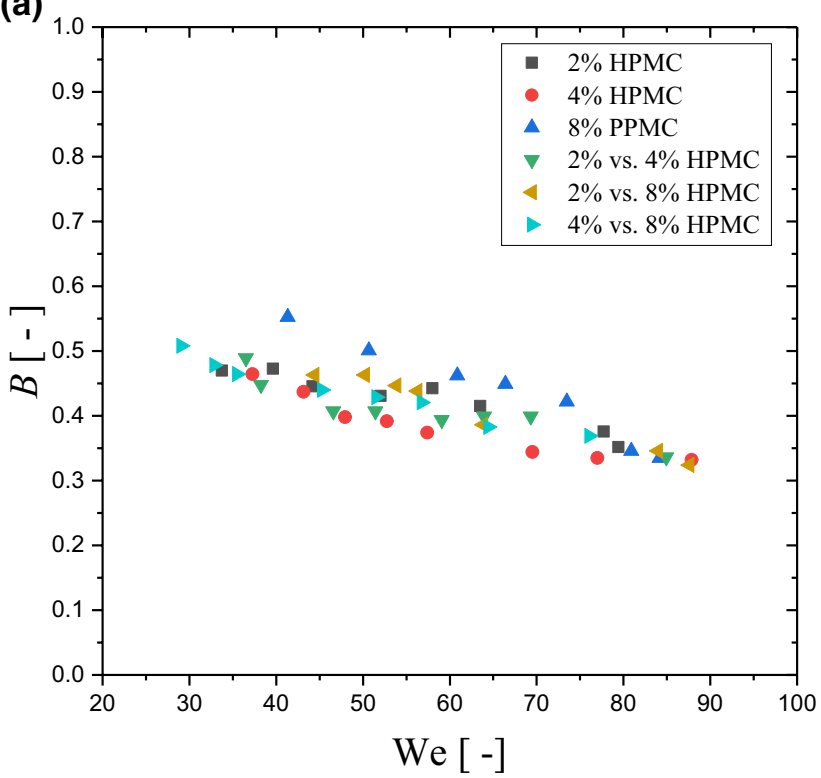

Fig. 15 Application of the model of Planchette et al. (2012) on the experimental data of the boundary of the stretching separation regime. Applying the model shows that the data collapse on

\subsubsection{Reflexive separation boundary}

The boundary of the reflexive separation regime is widely predicted by shifting the model of Ashgriz and Poo (1990), Eq. (13), towards higher We using Oh correlations (Sommerfeld and Pasternak 2019). The shifted model of Ashgriz and Poo (1990) is given by

$$
\begin{aligned}
\mathrm{We}_{\mathrm{c}}= & \left(\mathrm{We}_{\mathrm{FC} / \mathrm{RS}(f(\mathrm{Oh}))}-W e_{\mathrm{FC} / \mathrm{RS}(\text { water })}\right) \\
& +3\left[7\left(1+\Delta^{3}\right)^{\frac{2}{3}}-4\left(1+\Delta^{2}\right)\right] \frac{\Delta\left(1+\Delta^{3}\right)^{2}}{\Delta^{6} ¥_{1}+¥_{2}},
\end{aligned}
$$

where $\mathrm{We}_{\mathrm{FC} / \mathrm{RS}(f(\mathrm{O})}$ is the onset of the reflexive separation regime predicted by Oh correlations detailed in Sect. 2.3, Eqs. (16, 17 and 18), and $\mathrm{We}_{\mathrm{FC} / \mathrm{RS} \text { (water) }}$ is the onset of the reflexive separation of water which is equal to $6\left[7(2)^{\frac{2}{3}}-8\right]$ at $B=0$ from Eq. (13). $¥_{1}$, and $¥_{2}$ are defined in Eqs. (14 and $15)$.

However, the more detailed model, of Planchette et al. (2017), Eq. (20) can be used as an alternative to the Oh correlations and a comparison of the two approaches will be made [i.e., the model Planchette et al. (2017) vs. Oh correlations in Eqs. (17 and 18)]. Before implementing any model, it should be noted that in the case of collisions between droplets with different viscosities an Oh based on the arithmetic mean viscosity will be used. This is attributed to the observed intermediate value of $\mathrm{We}_{\mathrm{FC} / \mathrm{RS}}$ in the non-identical viscosity collisions compared to the identical collisions, in Sect. 4.2.3.

(b)

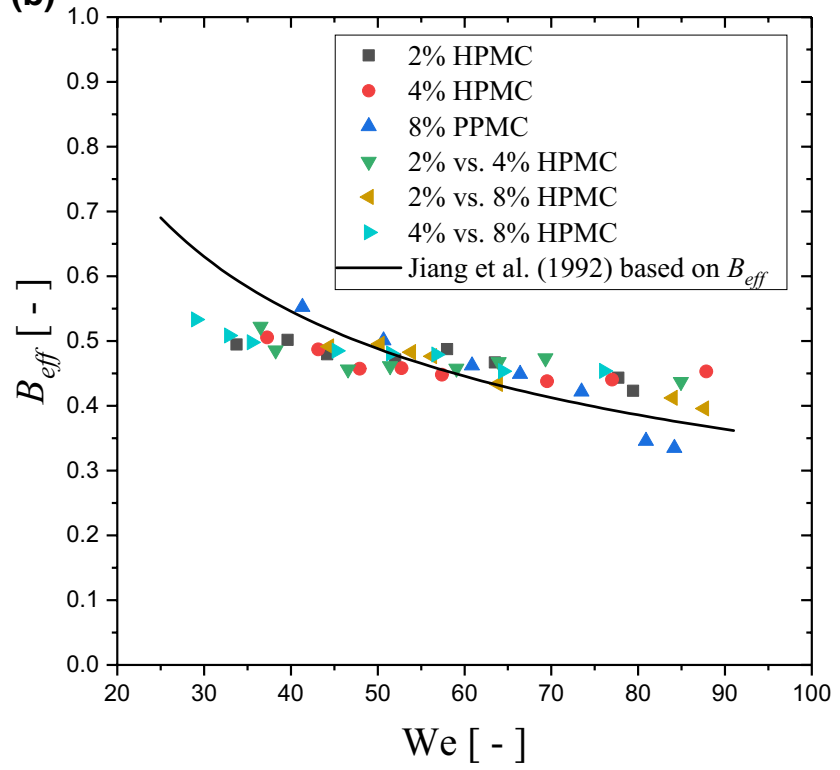

the boundary of the $8 \%$ HPMC (the reference boundary). a Original experimental data; $\mathbf{b}$ data plotted vs. the effective impact parameter $B_{\text {eff }}$ using the approach of Planchette et al. (2012), Eq. (12) 
The model of Planchette et al. (2017), Eq. (20), can be written in terms of We and $\mathrm{Oh}$ :

$\mathrm{We}_{\mathrm{FC} / \mathrm{RS}}=\left(0.8 p \zeta_{\text {crit }}\right)^{2} \frac{24 \sqrt{2} / \pi}{\left(1-a-q /\left(W e_{\mathrm{FC} / \mathrm{RS}}^{0.5} \mathrm{Oh}\right)\right.} \mathrm{Oh}$.

The model has three adjustable parameters $\zeta_{\text {crit }}, a$ and $q$. The loss ratio $a$ was experimentally determined for the three systems, using the same procedure used in Planchette et al. (2017). It was confirmed to be independent of the initial velocity, and the system viscosity, see supplementary material. Its measured values agree very well with those reported by Planchette et al. (2017) $(a \sim 0.67 \pm 0.02)$. $\zeta_{\text {crit }}$, the critical aspect ratio for break-up, was also set at 3 as in Planchette et al. (2017) breakage criteria. The model was used with $q=0.025(1-a)=0.00825$ and a pre-factor $p=2.38$ as in Planchette et al. (2017).

Figure 16 compares the $\mathrm{We}_{\mathrm{FC} / \mathrm{RS}}$ predictions of the two alternative models against each other and against the experimentally observed values. Similar trends are seen in both models; however, at higher Oh values, the gradient of the model of Planchette et al. (2017), Eq. (23), is lower and an improved prediction is seen vs. the correlations of Gotaas et al. (2007), Eqs. (17 and 18), which have some significant error. This error attributed to the noticeable scattering of the data when the Oh correlation was produced, the reader is referred to the review of Sommerfeld and Pasternak (2019). Both models show under-prediction in case of $2 \%$ HPMC, the reason behind this is unclear and further investigations for low concentration HPMC droplets would be of value especially for $\mathrm{Oh}<0.04$.

Based on the above, the model of Planchette shows promising performance in predicting the onset of reflexive separation for head-on collisions for both identical and nonidentical viscosity cases. It is, therefore, used as an alternative to the correlations of Gotaas et al. (2007) to predict the reflexive separation boundary using Eq. (22). The boundaries predicted by this approach are shown in Figs. 5 and 6 .

An alternative model that can be applied to predict the reflexive separation regime boundary is the model of $\mathrm{Hu}$ et al. (2017), Eq. (16). This model uses a fitting parameter $\alpha_{2}$ to represent the ratio of the entire viscous loss, to the impact kinetic energy. Therefore, in addition to the collisions of identical viscosities, this model can be implemented to predict the reflexive separation regime boundary for the collisions of droplets with non-identical viscosities. This is done by setting $\alpha_{2}$ to fit the predicted $\mathrm{We}_{\mathrm{FC} / \mathrm{RS}}$ to the experimentally measured boundary for head-on collision. The fitted viscous loss parameters $\alpha_{2}$, in the case of collisions of identical droplets, increase as the viscosity increases. It is 0.55 for $2 \%$ HPMC, and 0.75 for $4 \%$ HPMC. However, contrary to the stretching separation, the non-identical viscosity system $2 \%$ vs. $4 \%$ HPMC shows an intermediate viscous loss parameter, $\alpha_{2}=0.63$, compared to the identical droplets systems. This is consistent with the mixing between the colliding droplets as shown at the last set of images in Fig. 13.

The ratio of the viscous loss to the kinetic energy is expected to not change noticeably by changing the droplet viscosity. This is because at the onset of the reflexive separation, of identical droplets collisions, the separated droplets have a similar surface area to the initial droplets and possess negligible kinetic energy compared to the initial droplets. This is to say almost all kinetic energy has been lost. This was also commented on by Planchette et al. (2017). Therefore, the ratio of the total viscous loss to the initial kinetic energy is expected to be close to unity and higher than those of the model of $\mathrm{Hu}$ et al. (2017) and to not vary significantly by changing the viscosity of the droplets. This raises a need for more investigation into the assumptions of $\mathrm{Hu}$ et al. (2017), which is out of the scope of this paper.

Although the model of Hu et al. (2017) shows unphysical $\alpha_{2}$ values, it is still interesting to be used and compared with the shifted model of Ashgriz and Poo (1990). At $B>0$ both models show good agreement with the experimental data for the identical droplets 4\% HPMC, as shown in Fig. 5. However, the model of Hu et al. (2017) slightly over-predicts $B$ in case of $2 \%$ HPMC and $2 \%$ vs. $4 \%$ HPMC, whereas the model of Ashgriz and Poo (1990) shows good agreement in both cases, as shown in Figs. 5 and 6. This requires more investigation into the role of the impact parameter and its implementation in the modelling of the boundary of the reflexive separation regime.

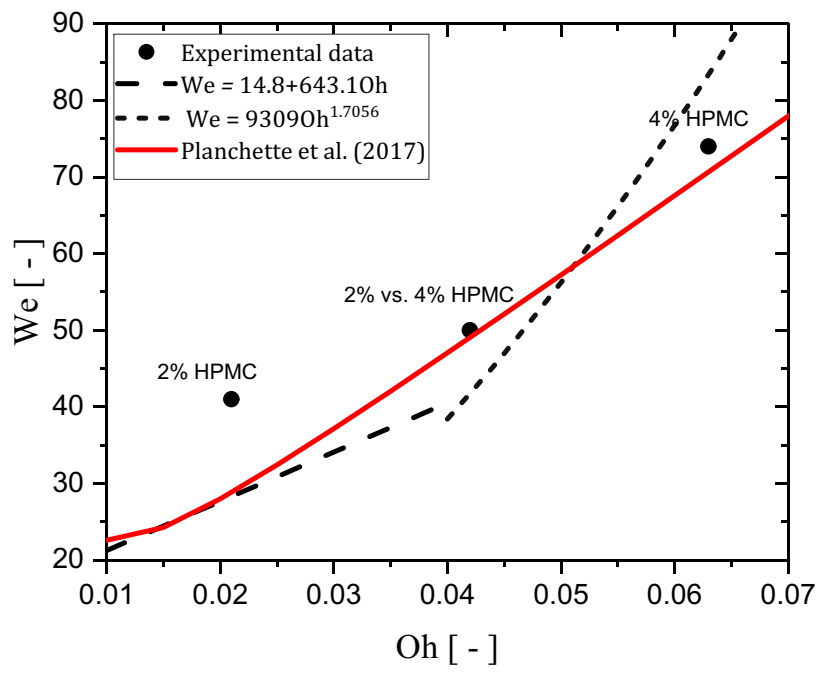

Fig. 16 Performance comparison between the model of Planchette et al. (2017) and the Oh correlations in predicting the onset of the reflexive separation regime at head-on collisions 


\section{Conclusions}

Viscosity is an important factor in determining the collision behaviour of droplets. This work has extended the understanding of the effects of viscosity by investigating collisions between miscible droplets of non-identical viscosity in which only the viscosity was significantly different. To provide a comparative basis for the non-identical viscosity collisions, the identical collision regime maps, reported in Al-Dirawi and Bayly (2019), were used and analysis of these extended across the whole of the collision space (rather than the previous focus on bouncing). For the range of viscosities studied we found similar behaviours to the identical droplets, with the same regimes identified in the We, $B$ space and clear boundaries between regimes that were shifted vs. the identical cases. The mechanisms involved in shifting the boundaries are slightly different in each case, and depend on the role of viscosity on the transfer of collision energy to deformation and then its effect on the subsequent separation mechanism. The following conclusions can be drawn for each boundary.

\subsection{Bouncing transition}

Increasing the viscosity promotes fast coalescence by suppressing the bouncing regime due to the higher viscosity decreasing the droplet deformation and allowing more rapid drainage of the air layer between the colliding droplets. The transition from bouncing to fast coalescence, therefore, occurs at lower We. In the non-identical case this transition occurs at an intermediate value between the transition We values of the two identical cases at the lower and higher viscosity. In comparison to identical collisions at the lower viscosity, the deformation is only reduced in one of the droplets; therefore, the drainage of the air layer only benefits from half the change vs. the collisions at the higher viscosity. A new phenomenon, partial bouncing, is also observed at high viscosity ratio, whereby a thin ligament between the bounced droplets is observed. This ligament separates from the higher viscosity droplet and retracts to the lower viscosity droplet. The same modelling framework developed in our previous work can be used to fit the regime boundaries of collisions of droplets with non-identical viscosities and changes in the loss factor $(\alpha)$ and the shape factor are consistent with the theory.

\subsection{Stretching separation transition}

In identical collisions, at the higher Oh studied, increasing the viscosity shifts the boundary of the stretching separation regime to higher $B$ values this might be anticipated due to the increase in the viscous loss and the relative importance of the viscosity term in the Oh number. However, at low $\mathrm{Oh}<0.1$ increasing the viscosity showed the opposite behaviour, this may be due to the increased role of surface tension. The reasons are not entirely clear and more investigation into this behaviour is required. In the case of non-identical collisions, we see the stretching separation regime boundaries remaining very similar to the identical case for lower viscosity droplets. This was shown to be due to the filament being drawn mainly from the lower viscosity droplet and consequently its break-up occurring in this low viscosity region. In addition, the satellite droplets produced from the break-up of the ligament during stretching separation, have a similar composition to the lower viscosity droplet, with only a small amount of mixing. Consequently, the adjustable parameters in the model of Jiang et al. (1992) were very similar to those of the lower viscosity case. The model of Planchette et al. (2012) was also evaluated and the use of an effective impact parameter allowed the collapse of the experimental data to a unified curve. However, its value was limited as no clear relationship between the $U^{*}$ fitting parameter and the physical properties of the droplets was observed.

\subsection{Reflexive separation transition}

For identical droplets, the transition from fast coalescence to reflexive separation is shifted to higher We due to increased viscous loss in the stretching and reflexive motion of the droplets. For non-identical drops, a similar trend is seen, with the transition moving to intermediate values of $\mathrm{We}$ compared to the identical cases. Deformation is reduced and partial mixing is seen between the colliding droplets so there is both less energy in the reflexive separation and intermediate viscosity in the necking ligament. Interestingly because of the mixing, a concentration gradient is setup and the lower viscosity droplet detaches at the low viscosity end of the ligament. This leads to a smaller low viscosity droplet and a larger droplet composed of both the higher viscosity fluid mixed with a little lower viscosity fluid. The application of the model of Ashgriz and Poo (1990), using an offset based on a prediction of the head-on boundary $\mathrm{We}_{\mathrm{FC} / \mathrm{RS}}$ using the approach of Planchette et al. (2017) can be used for both the identical and the non-identical cases based on the average $\mathrm{Oh}$. It was found to give slightly better predictions than the correlation proposed by Gotaas et al. (2007) in the higher Oh systems tested. Neither model gave a good prediction of the onset of the reflexive separation at head-on for 2\% HPMC, the lowest Oh system. The model of Hu et al. (2017) was found to give an approximate fit to the reflexive separation boundary of the non-identical case when an intermediate loss factor was used. However, at $B>0$, the fit of this model was found to be poorer than that of the model of Ashgriz and Poo (1990). Moreover, the model of Hu et al. (2017) shows unphysical viscous loss factors. Therefore, further work is 
required to understand the model's assumptions related to the role of impact parameter and the viscously lost energy.

This work represents an initial step in characterising these non-identical collisions. Several phenomena have been observed and mechanistic insights obtained; however, there are clearly opportunities to learn more about these interesting, industrially relevant systems.

\section{Supplementary material}

See supplementary material for data on the viscous loss vs. kinetic energy during the compressive dynamics at head-on collisions of identical droplets.

Acknowledgements The authors gratefully acknowledge the useful discussions and assistance of Prof Phil Threlfall-Holmes, Prof Nik Kapur Khaled Al-Ghaithi, Thomas C. Sykes, and Gurdev Bhogal from the University of Leeds. The work was supported by EPSRC Project: 'Evaporative Drying of Droplets and the Formation of Micro-structured and Functional Particles and Films' (Grant Ref: EP/N025245/1) and the University of Leeds.

Open Access This article is licensed under a Creative Commons Attribution 4.0 International License, which permits use, sharing, adaptation, distribution and reproduction in any medium or format, as long as you give appropriate credit to the original author(s) and the source, provide a link to the Creative Commons licence, and indicate if changes were made. The images or other third party material in this article are included in the article's Creative Commons licence, unless indicated otherwise in a credit line to the material. If material is not included in the article's Creative Commons licence and your intended use is not permitted by statutory regulation or exceeds the permitted use, you will need to obtain permission directly from the copyright holder. To view a copy of this licence, visit http://creativecommons.org/licenses/by/4.0/.

\section{References}

Al-Dirawi KH, Bayly AE (2019) A new model for the bouncing regime boundary in binary droplet collisions. Phys Fluids 31(2):027105

Ashgriz N, Poo JY (1990) Coalescence and separation in binary collisions of liquid drops. J Fluid Mech 221:183-204

Basu AS (2013) Droplet morphometry and velocimetry (DMV): a video processing software for time-resolved, label-free tracking of droplet parameters. Lab Chip 13(10):1892-1901

Brazier-Smith PR, Jennings SG, Latham J (1972) The interaction of falling water drops: coalescence. Proc R Soc Lond A Math Phys Sci 326(1566):393-408

Chen RH (2007) Diesel-diesel and diesel-ethanol drop collisions. Appl Therm Eng 27(2-3):604-610

Chen RH, Chen CT (2006) Collision between immiscible drops with large surface tension difference: diesel oil and water. Exp Fluids 41(3):453-461

Estrade JP, Carentz H, Lavergne G, Biscos Y (1999) Experimental investigation of dynamic binary collision of ethanol droplets-a model for droplet coalescence and bouncing. Int J Heat Fluid Flow 20(5):486-491
Finotello G, Padding JT, Deen NG, Jongsma A, Innings F, Kuipers JAM (2017) Effect of viscosity on droplet-droplet collisional interaction. Phys Fluids 29(6):067102

Finotello G, De S, Vrouwenvelder JC, Padding JT, Buist KA, Jongsma A, Innings F, Kuipers JAM (2018a) Experimental investigation of non-Newtonian droplet collisions: the role of extensional viscosity. Exp Fluids 59(7):113

Finotello G, Kooiman RF, Padding JT, Buist KA, Jongsma A, Innings F, Kuipers JAM (2018b) The dynamics of milk droplet-droplet collisions. Exp Fluids 59(1):17

Focke C, Kuschel M, Sommerfeld M, Bothe D (2013) Collision between high and low viscosity droplets: direct numerical simulations and experiments. Int J Multiph Flow 56:81-92

Francia V, Martín L, Bayly AE, Simmons MJ (2016) Agglomeration in counter-current spray drying towers. Part A: particle growth and the effect of nozzle height. Powder Technol 301:1330-1343

Gao TC, Chen RH, Pu JY, Lin TH (2005) Collision between an ethanol drop and a water drop. Exp Fluids 38(6):731-738

Gotaas C, Havelka P, Jakobsen HA, Svendsen HF, Hase M, Roth N, Weigand B (2007) Effect of viscosity on droplet-droplet collision outcome. Exp Study Numer Comp Phys Fluids 19(10): 102106

Hicks PD, Purvis R (2010) Air cushioning and bubble entrapment in three-dimensional droplet impacts. J Fluid Mech 649:135-163

Hu C, Xia S, Li C, Wu G (2017) Three-dimensional numerical investigation and modeling of binary alumina droplet collisions. Int $\mathrm{J}$ Heat Mass Transf 113:569-588

Jiang YJ, Umemura A, Law CK (1992) An experimental investigation on the collision behaviour of hydrocarbon droplets. J Fluid Mech 234:171-190

Kolinski JM, Rubinstein SM, Mandre S, Brenner MP, Weitz DA, Mahadevan L (2012) Skating on a film of air: drops impacting on a surface. Phys Rev Lett 108(7):074503

Krishnan KG, Loth E (2015) Effects of gas and droplet characteristics on drop-drop collision outcome regimes. Int J Multiph Flow 77:171-186

Kuschel M, Sommerfeld M (2013) Investigation of droplet collisions for solutions with different solids content. Exp Fluids 54(2):1440

Langley K, Li EQ, Thoroddsen ST (2017) Impact of ultra-viscous drops: air-film gliding and extreme wetting. J Fluid Mech 813:647-666

McKinley GH (2005) Dimensionless groups for understanding free surface flows of complex fluids. Soc Rheol (Rheol Bull) 74(2):6-9

Ohnesorge WV (1936) Die bildung von tropfen an düsen und die auflösung flüssiger strahlen. ZAMM J Appl Math Mech 16(6):355-358

Orme M (1997) Experiments on droplet collisions, bounce, coalescence and disruption. Prog Energy Combust Sci 23(1):65-79

Pan KL, Law CK, Zhou B (2008) Experimental and mechanistic description ofmerging and bouncing in head-on binary droplet collision. J Appl Phy 103(6):064901

Pan KL, Tseng YH, Chen JC, Huang KL, Wang CH, Lai MC (2016) Controlling droplet bouncing and coalescence with surfactant. J Fluid Mech 799:603-636

Planchette C, Lorenceau E, Brenn G (2010) Liquid encapsulation by binary collisions of immiscible liquid drops. Colloids Surf A Physicochem Eng Asp 365(1-3):89-94

Planchette C, Lorenceau E, Brenn G (2011) Binary collisions of immiscible liquid drops for liquid encapsulation. Fluid Dyn Mater Process 7(3):279-301

Planchette C, Lorenceau E, Brenn G (2012) The onset of fragmentation in binary liquid drop collisions. J Fluid Mech 702:5-25

Planchette C, Hinterbichler H, Liu M, Bothe D, Brenn G (2017) Colliding drops as coalescing and fragmenting liquid springs. J Fluid Mech 814:277-300 
Post SL, Abraham J (2002) Modeling the outcome of drop-drop collisions in Diesel sprays. Int J Multiph Flow 28(6):997-1019

Qian J, Law CK (1997) Regimes of coalescence and separation in droplet collision. J Fluid Mech 331:59-80

Sommerfeld M, Kuschel M (2016) Modelling droplet collision outcomes for different substances and viscosities. Exp Fluids 57(12): 187

Sommerfeld M, Pasternak L (2019) Advances in modelling of binary droplet collision outcomes in sprays: a review of available knowledge. Int J Multip Flow 117:182-205

Southwell DB, Langrish TAG (2000) Observations of flow patterns in a spray dryer. Dry Technol 18(3):661-685

Tsuru D, Tajima H, Ishibashi R, Kawauchi S (2010) Droplet collision modelling between merging immiscible sprays in direct water injection system. In: ILASS Europe, 23rd annual conference on liquid atomization and spray systems, Brno, Czech Republic
Verdurmen REM, Menn P, Ritzert J, Blei S, Nhumaio GCS, Sonne Sørensen T, Gunsing M, Straatsma J, Verschueren M, Sibeijn M, Schulte G (2004) Simulation of agglomeration in spray drying installations: the EDECAD project. Dry Technol 22(6):1403-1461

Willis K, Orme M (2003) Binary droplet collisions in a vacuum environment: an experimental investigation of the role of viscosity. Exp Fluids 34(1):28-41

Publisher's Note Springer Nature remains neutral with regard to jurisdictional claims in published maps and institutional affiliations. 